\title{
A Poisson Algebra for Abelian Yang-Mills Fields on Riemannian Manifolds with Boundary
}

\author{
Homero G. Díaz-Marín † \\ Facultad de Ciencias Físico-Matemáticas, Universidad Michoacana de San Nicolás de Hidalgo, \\ Morelia C.P. 58060, Mexico; hdiaz@umich.mx \\ † Current address: Ciudad Universitaria, Morelia C.P. 58060, Michoacán, México.
}

Received: 28 May 2019; Accepted: 4 June 2019; Published: 4 July 2019

\begin{abstract}
We define a family of observables for abelian Yang-Mills fields associated to compact regions $U \subseteq M$ with smooth boundary in Riemannian manifolds. Each observable is parametrized by a first variation of solutions and arises as the integration of gauge invariant conserved current along admissible hypersurfaces contained in the region. The Poisson bracket uses the integration of a canonical multisymplectic current.
\end{abstract}

Keywords: Yang-Mills gauge fields; variational bicomplex; riemannian manifold; conserved currents

MSC: 70S10; 70S15; 58Z05; 49S05

\section{Introduction}

In Classical Covariant Field Theory two desirable conditions are required for a family of observables: In one side we require this function to separate solutions of the Euler-Lagrange equations. On the other hand, we need the Jacobi identity in order to have a Lie (Poisson) bracket. It is a known problem to characterize those theories accomplishing these two requirements, as pointed out in $[1,2]$ and others. There are two main difficulties. On one hand, under locality assumptions, Jacobi identity is well established but generically there are few observables associated with conservation laws given by Noether's First Theorem, see for instance [3]. On the other hand, extending to non-locality of variations of solutions, we may provide enough observables, see for instance $[4,5]$, nevertheless the Jacobi identity does not necessarily hold, see [6].

For linear theories there are no such difficulties, and vector fields in the space of solutions can be modeled as in Theorem 2, see also [7]. For instance, in Lorentzian globally hyperbolic spacetimes, Maxwell equations [8] exhibit a family of observables, related to the Aharomov-Bohm effect, and a Poisson bracket constructed with Peierls method for local variables. We provide a similar set of observables for the abelian Yang-Mills (YM) fields on Riemannian manifolds. This could be mentioned as the novelty introduced in this work, although our aim is to prepare the scenario for non-abelian (non-linear) YM fields. We adopt the Lagrangian approach of the variational bicomplex formalism, see [9-11] rather than the Hamiltonian multysimplectic formalism approach to describe non abelian YM fields, see [12,13].

We consider regions $U$ with smooth boundary $\partial U$ both contained in a $n$-dimensional Riemannian manifold, usually $n=4$. Here we avoid the complications of corners in $\partial U$ which will be treated elsewhere. For a principal bundle we take solutions of the Yang-Mills (YM) equations for the abelian $U(1)$ structure group. We are interested in defining a family of observables for YM solutions in $U$, $\eta \in \mathcal{A}_{U}$, of the integral form

$$
f_{\Sigma}(\eta)=\int_{\Sigma} \mathrm{j} \eta^{*} F
$$


defined for a 3-dimensional compact Riemannian admissible smooth hypersurface $i_{\Sigma}: \Sigma \longleftrightarrow U$ with volume form $v_{\Sigma}$, where admissibility means $\partial \Sigma \subseteq \partial U$, see [14]. Observable currents, are horizontal $(n-1)$-forms, $F \in \Omega^{n-1,0}\left(\left.J Y\right|_{U}\right)$, in the $\infty$ - jet bundle $J Y$ associated to sections of the affine bundle $Y \rightarrow M$ of connections. The local invariance condition is then assumed by imposing $\left.d_{h} F\right|_{U}=0$, when restricted to the locus of the $Y M$ equations $\mathcal{E}_{L} \cdot d_{h}$ is the horizontal differential, see the notation of the variational bicomplex formalism in Appendix A. We adapt helicity for hypersurfaces embedded properly in general compact regions $U$, rather than considering cylinder regions with space-like slices, $\Sigma \times\left[t_{1}, t_{2}\right]$, this is related to the General Boundary Formalism for field theories, see [15] and references therein.

The idea is to define the relative helicity from hydrodynamics properly adapted to YM fields as a local observable. In order to motivate this definition we recall the notion of helicity from magneto-hydrodynamics. For a divergence (non-autonomous) free vector field, $\xi=\xi(t) \in \mathfrak{X}_{\partial \Sigma}(\Sigma)$ in a three-dimensional Riemannian manifold $\Sigma$ tangent to the boundary $\partial \Sigma$, helicity is defined as

$$
\int_{\Sigma} \bar{g}(v, \xi) v_{\Sigma}
$$

where one considers the vector field $v=v(t)$, as a potential in $\Sigma$. Helicity of $\xi$ measures globally the degree of self-linking of its flow. Helicity remains an invariant for every $v_{\Sigma}$-preserving diffeomorphism of $\Sigma$ that carries the boundary $\partial \Sigma$ into itself, where $v_{\Sigma}$ is given by the volume form on $\Sigma$. The situation can be dually described in terms of 1-forms. If $\alpha=\bar{g}(v, \cdot)$ where $\bar{g}$ is the Riemannian metric on $\Sigma$, then under the additional topological condition, $H_{\mathrm{dR}}^{2}(\Sigma)=0$, there exists a potential $\alpha \in \Omega^{1}(\Sigma)$ such that $d \alpha=\iota_{\xi} v_{\Sigma}$. Here helicity reads as

$$
\int_{\Sigma} \alpha \wedge d \alpha
$$

It does depend just on the vorticity $d \alpha$ although for its definition the potential 1-form $\alpha$ or the vector field $v$, respectively, may intervene.

If we adopt $v \in \mathfrak{X}_{\partial \Sigma}(\Sigma)$ divergence-free or $d^{\star \Sigma}(\alpha)=0$, respectively, then the property of isovorticity holds for $v(t)$ for the magnetic potential, as well as for any solution of the Euler equation of hydrodynamics. This means that $\xi\left(t_{2}\right)$ can be constructed as the image of $\xi\left(t_{1}\right)$ under a diffeomorphism and if we consider a space-time domain $\Sigma \times\left[t_{1}, t_{2}\right]$, then helicity does not depend on the parameter $t$ of the non-autonomous flow. To review this concepts see for instance [16,17].

Under the assumption of simple connectednes of $\Sigma$, then the Lie algebra of divergence-free vector fields, have a bilinear form, relative helicity, defined as

$$
[\alpha, \beta]_{\Sigma}=\int_{\Sigma} d \alpha \wedge \beta
$$

Notice that helicity is $[\alpha, \alpha]_{\Sigma}$ and also that $[\cdot, \cdot]_{\Sigma}$ is a symmetric bilinear form under the assumption of closedness for $\Sigma$.

Considering YM solutions $\eta=\eta_{0}+\varphi \in \mathcal{A}_{U}$, where $\eta_{0} \in \mathcal{A}_{U}$ is a fixed connection and $\varphi=\eta-\eta_{0}$ is a 1-form in $M$, we would like to define the field strength helicity as in (2). Choose a tubular neighborhood $\Sigma_{\varepsilon} \subseteq U$ of $\Sigma^{0}:=i_{\Sigma}(\Sigma, \tau)$ with exponential coordinates $X_{\Sigma}: \Sigma \times[-\varepsilon, \varepsilon] \rightarrow \Sigma_{\varepsilon}$, with embedding $i_{\Sigma}=X_{\Sigma}(\cdot, 0)$. We take $\tilde{\varphi}=\varphi+\left.d f\right|_{\Sigma_{\varepsilon}}$ an axial gauge fixing, that is a 1-form such that in $\Sigma^{0}$ has no normal component. In addition, we may suppose that $\psi_{\eta}^{\Sigma^{0}}=i_{\Sigma}^{*} \tilde{\varphi}$, as well as $\left.\frac{d}{d \tau}\right|_{\tau=0} \psi_{\eta}^{\Sigma^{\tau}}$ are divergence-free. See Appendix on the geometry of abelian YM fields in [15].

Then the helicity for abelian YM fields could be defined as

$$
[\tilde{\varphi}, \tilde{\varphi}]_{\Sigma}=\left.\int_{\Sigma} \psi_{\eta}^{\Sigma^{0}} \wedge \star_{\Sigma} \frac{d \psi_{\eta}^{\Sigma^{\tau}}}{d \tau}\right|_{\tau=0},
$$


where $\star_{\Sigma}$ is the Hodge operator associated to the induced Riemannian metric $\bar{g}$ on $\Sigma$. Hence we could define helicity as in (1) for the vector fields $v, \xi$ defined as $\bar{g}(v, \cdot)=\psi_{\eta}^{\Sigma^{0}}, \quad \bar{g}(\xi, \cdot)=\left.\frac{d \psi_{\eta}^{\Sigma \tau}}{d \tau}\right|_{\tau=0}$.

Nevertheless, this notion of helicity would depend on the gauge fixing choice, therefore cannot be generalized as a gauge invariant observable. Moreover, we do not get a local $\mathrm{d}_{\mathrm{h}}$-closedness condition for an observable current: if $U^{\prime} \subseteq U$ is an open region such that $\partial U^{\prime}=\Sigma-\Sigma^{\prime}$, then

$$
[\tilde{\varphi}, \tilde{\varphi}]_{\Sigma}=[\tilde{\varphi}, \tilde{\varphi}]_{\Sigma^{\prime}}+\int_{U^{\prime}} L(\mathrm{j} \eta)
$$

where $L$ is the Lagrangian density. We will rather try to define the relative helicity of YM fields. Take $\eta^{\prime}=\eta_{0}+\varphi^{\prime} \in \mathcal{A}_{U}$ any other solution. Take a first variation of solutions $\varphi$, let us define

$$
\left[\varphi, \varphi^{\prime}\right]_{\Sigma}=\int_{\Sigma} i_{\Sigma}^{*}\left(\varphi^{\prime} \wedge \star d \varphi\right) .
$$

Then for gauge translations $\eta^{\prime}+d f$ we would have $\left[\varphi, \varphi^{\prime}\right]_{\Sigma}=\left[\varphi, \varphi^{\prime}+d f\right]_{\Sigma}$. Moreover, if $U^{\prime} \subseteq U$ is an open region such that $\partial U^{\prime}=\Sigma-\Sigma^{\prime}$, then $\left[\varphi, \varphi^{\prime}\right]_{\Sigma}=\left[\varphi, \varphi^{\prime}\right]_{\Sigma^{\prime}}$. Thus for every couple $\eta, \varphi$ where $\eta \in \mathcal{A}_{U}$ and $\varphi$ is a first variations of solutions, we consider the antisymmetric component of the relative helicity or simply $\varphi$-helicity,

$$
h_{\Sigma}^{\varphi}\left(\eta^{\prime}\right):=\frac{1}{2}\left(\left[\varphi, \varphi^{\prime}\right]_{\Sigma}-\left[\varphi^{\prime}, \varphi\right]_{\Sigma}\right) .
$$

In Section 4 we formalize this construction in the language of the variational bicomplex, see Appendix A.

\section{Variational Bicomplex Formalism for Abelian YM Fields}

Along this section we adopt the terminology and notation of the variational bicomplex formalism, for the readers convenience we give a brief presentation and references for this in Appendix A. Let $\mathcal{P} \rightarrow M$ be a principal bundle on a Riemannian manifold $(M, g)$ with structure group $G=U(1)$ and $U \subseteq M$ a region with smooth boundary. Let $\pi: Y \rightarrow M$ with $Y=J^{1} \mathcal{P} / G$ be the affine bundle whose sections $\Gamma(Y)$ are the $G$-covariant connections on $\mathcal{P}$.

For abelian YM, the Lagrangian density $L=\mathrm{L} v \in \Omega^{n, 0}(\mathrm{~J} Y)$ is defined by the Lagrangian

$$
\mathrm{L}=\frac{1}{4} \sum_{i, j=1}^{n} \omega^{i j}\left(A_{i}^{j}-A_{j}^{i}\right)^{2}
$$

where this expresion corresponds to local coordinates $\left(x_{1}, \ldots, x_{n} ; A^{1}, \ldots, A^{n} ; A_{j}^{i}\right)$ in $\mathrm{J}^{1} Y, v=d x^{1} \wedge$ $\cdots \wedge d x^{n}$ is a fixed volume form in the base and $\omega^{i j}=\sqrt{|\operatorname{det} g|} g^{i i} g j$, with $g_{i j}$ the Riemannian metric in $U$.

Then $E(L)=\sum_{i=1}^{n} E_{i}(\mathrm{~L}) \vartheta^{i} \wedge v$ denote the Euler-Lagrange equations, where $\vartheta^{i}=\mathrm{d}_{\mathrm{v}} A^{i}$ stands for the basis for the vertical 1 -forms in $J^{1} Y$. Thus $Y M$ equations have locus which is the prolongation $\mathcal{E}_{L} \subseteq J Y$ of $\{E(L)=0\} \subseteq J^{2} Y$. In the local coordinate chart,

$$
E_{i}(\mathrm{~L})=\sum_{j=1}^{n} \frac{\mathrm{d}}{\mathrm{d} x^{j}}\left(\omega^{i j}\left(A_{i}^{j}-A_{j}^{i}\right)\right)=0, \quad \forall i=1, \ldots, n .
$$

The space of solutions over $U$ is

$$
\mathcal{A}_{U}=\left\{\eta \in \Gamma\left(\left.Y\right|_{U}\right): \mathrm{j} \eta(x) \in \mathcal{E}_{L}\right\}
$$

Thus solutions $\eta$ satisfy $j \eta^{*} E(L)=0$. 
The linearized equations for any (local) evolutionary vector field, $V \in \mathfrak{E v}(J Y)$, are

$$
\left.\mathrm{I}\left(\mathrm{d}_{\mathrm{v}} \iota_{j V} E(L)\right)\right|_{\mathcal{E}_{L}}=0
$$

where I $: \Omega^{n, k}(J Y) \rightarrow \Omega^{n, k}(J Y)$ is the integration by parts operator, see its definition in [18]. In local coordinates this linearized equation reads as

$$
\left.\sum_{j=1}^{n} \frac{\mathrm{d}}{\mathrm{d} x^{j}}\left(\omega^{i j}\left(\frac{\mathrm{d}}{\mathrm{d} x^{i}} V^{j}-\frac{\mathrm{d}}{\mathrm{d} x^{j}} V^{i}\right)\right)\right|_{\mathcal{E}_{L}}=0, \quad \forall i=1, \ldots, n, V=\sum_{i=1}^{n} V^{i} \frac{\partial}{\partial A^{i}} .
$$

Let $\mathfrak{F}_{U} \subseteq \mathfrak{E} \mathfrak{v}(J Y)$ be the Lie subalgebra of those evolutionary vector fields satisfying the linearized Euler-Lagrange equations. The Lie algebra $\mathfrak{F}_{U}$ will turn out to be our model for variations of $Y M$ solutions. For example, the radial evolutionary vector field $R=\sum_{a} A^{a} \frac{\partial}{\partial A^{a}}$ whose prolongation is

$$
\mathrm{j} R=\sum_{a} A^{a} \frac{\partial}{\partial A^{a}}+\sum_{i} A_{i}^{a} \frac{\partial}{\partial A_{i}^{a}}+\ldots
$$

is a symmetry of the YM PDE, i.e. $R \in \mathfrak{F}_{U}$. This is a general constructions of symmetries for linear PDEs, see [5].

The presymplectic current

$$
\Omega_{L}=\sum_{i, j=1}^{n} \omega^{i j}\left(\vartheta_{j}^{i}-\vartheta_{i}^{j}\right) \wedge \vartheta^{j} \wedge v^{i}
$$

with $d x^{i} \wedge v^{i}=v$, has the property stated in the following general Lemma.

Lemma 1 (Multysimplectic formula). For every $V, W \in \mathfrak{F} u$ we have

$$
\left.\mathrm{d}_{\mathrm{h}}\left(\iota_{\mathrm{j} W} \iota_{\mathrm{j} V} \Omega_{L}\right)\right|_{\mathcal{E}_{L}}=0
$$

Definition 1 (Gauge).

1. Those first variations of solutions $V \in \mathfrak{F}_{U}$ satisfying

$$
\left.\iota_{\mathrm{j} W} \mathcal{L}_{\mathrm{j} V} \Omega_{L}\right|_{\mathcal{E}_{L}}=\left.\iota_{\mathrm{j} W} \mathrm{~d}_{\mathrm{v}}\left(\iota_{\mathrm{j} V} \Omega_{L}\right)\right|_{\mathcal{E}_{L}}=\iota_{\mathrm{j} W} \mathrm{~d}_{\mathrm{h}} \sigma^{V}, \quad \forall W \in \mathfrak{F}_{U},
$$

define the Lie subalgebra of locally Hamiltonian first variations as $\hat{\mathfrak{F}}_{U}^{\mathrm{LH}} \subseteq \mathfrak{F}_{U}$.

2. We define the Lie algebra $\hat{\mathfrak{G}}_{U}$ of gauge first variations as those $X \in \mathfrak{F}_{U}$ satisfying locally the presymplectic degeneracy condition, i.e.,

$$
\left.\iota_{\mathrm{j} W}\left(\iota_{\mathrm{j} X} \Omega_{L}\right)\right|_{\mathcal{E}_{L}}=\iota_{\mathrm{j} W} \mathrm{~d}_{\mathrm{h}} \rho^{X}, \quad \forall W \in \mathfrak{F}_{U}
$$

For instance, the radial vector $R \in \mathfrak{F}_{U}$ defined in (6) is not locally hamiltonian, since it satisfies the Liouville condition $\iota_{j R} d_{v} \Omega_{L}=\Omega_{L}$, rather than condition (7).

In the second part of Definition 1 we may also have adopted $X \in \mathfrak{E} \mathfrak{v}(J Y)$ instead of $X \in \mathfrak{F}_{U}$ and

$$
\left.\iota_{\mathrm{j} W}\left(\iota_{\mathrm{j} X} \Omega_{L}\right)\right|_{\mathcal{E}_{L}}=\iota_{\mathrm{j} W} \mathrm{~d}_{\mathrm{h}} \rho^{X}, \quad \forall W \in \mathfrak{E v}\left(\left.J Y\right|_{U}\right)
$$

as is stated in the following assertion.

Proposition 1. Suppose that $V \in \mathfrak{E} \mathfrak{v}\left(\left.J Y\right|_{U}\right)$ satisfies

$$
\left.\iota_{\mathrm{j} W} \mathrm{~d}_{\mathrm{h}}\left(\iota_{\mathrm{j} V} \Omega_{L}\right)\right|_{\mathcal{E}_{L}}=0
$$


for every variation of solutions $W \in \mathfrak{F}_{U}$. Then $V \in \mathfrak{F}_{U}$.

Notice that the locally Hamiltonian condition is stronger than the property exhibited in Proposition 1 for every variation of solutions. Thus $\hat{\mathfrak{G}}_{U} \subseteq \hat{\mathfrak{F}}_{U}^{\mathrm{LH}}$.

Lemma 2. $\hat{\mathfrak{G}}_{U} \subseteq \hat{\mathfrak{F}}_{U}^{\mathrm{LH}}$ is a Lie ideal.

Proof. If $X, X^{\prime} \in \hat{\mathfrak{G}}_{U}$ then

$$
\iota_{\mathrm{j}\left[X, X^{\prime}\right]} \Omega_{L}=\mathrm{d}_{\mathrm{v}} \iota_{\mathrm{j} X} \iota_{\mathrm{j} X^{\prime}} \Omega_{L} \pm \iota_{\mathrm{j} X} \mathrm{~d}_{\mathrm{v}} \iota_{\mathrm{j} X^{\prime}} \Omega_{L} \pm \iota_{\mathrm{j} X^{\prime}} \mathrm{d}_{\mathrm{v}} \iota_{\mathrm{j} X} \Omega_{L}
$$

which by hypothesis and by anticommutativity of $d_{v} d_{h}=-d_{h} d_{v}$ is dh-exact, hence $\left[X, X^{\prime}\right] \in \hat{\mathfrak{G}}_{U}$ and therefore $\hat{\mathfrak{G}}_{U} \subseteq \mathfrak{F}_{U}$ is a Lie subalgebra. To see that $\hat{\mathfrak{G}}_{U} \subseteq \widehat{\mathfrak{F}}_{U}^{\mathrm{LH}}$, apply vertical derivation to (8).

Take $V \in \widehat{\mathfrak{F}}_{U}^{\mathrm{LH}}$, then $[V, X]$ apply vertical derivation to Equation (9) with $V=X^{\prime}$ and the condition of $d_{h}$-exactness for $\iota_{j X} d_{v} \iota_{j} \Omega_{L}$ implies the $d_{h}$-exactness of $\iota_{j}[X, V] \Omega_{L}$ holds. Therefore $[V, X] \in \hat{\mathfrak{G}}_{U}$.

Form Proposition 1 it follows also the following assertion.

Lemma 3. $\hat{\mathfrak{G}}_{U} \subseteq \mathfrak{E} \mathfrak{v}\left(\left.J Y\right|_{U}\right)$ is a Lie ideal, hence $\hat{\mathfrak{F}}_{U}^{\mathrm{LH}} / \hat{\mathfrak{G}}_{U} \subseteq \mathfrak{E v}\left(\left.J Y\right|_{U}\right) / \hat{\mathfrak{G}}_{U}$.

Lemma 4. If for every $W \in \mathfrak{F}_{U}$

$$
\left.\iota_{\mathrm{j} W}\left(\iota_{\mathrm{j} X} \Omega_{L}\right)\right|_{\mathcal{E}_{L}}=\left.\iota_{\mathrm{j} W} \mathrm{~d}_{\mathrm{h}} \rho^{X}\right|_{\mathcal{E}_{L^{\prime}}} \quad \rho^{X}=\sum_{i, j=1}^{n} \rho^{i j} \vartheta_{i}^{j} \wedge v^{j i} .
$$

holds, then in local coordinates $\omega^{i j} X^{j}=\frac{\mathrm{d}}{\mathrm{d} x^{j}} \rho^{i j}$ holds in $\mathcal{E}_{L}$ for each $i, j=1, \ldots, n$, where $d x^{j} \wedge v^{j i}=v^{i}$.

Definition 2 (Gauge with boundary condition).

1. The Lie subalgebra

$$
\mathfrak{F}_{U}^{\mathrm{LH}} \subseteq \hat{\mathfrak{F}}_{U}^{\mathrm{LH}}
$$

of locally Hamiltonian first variations with null boundary conditions, consists of those $V \in \hat{\mathfrak{F}}_{U}^{\mathrm{LH}}$ satisfying (7) and

$$
\left.\sigma^{V}\right|_{\partial U}=\left.\mathrm{d}_{\mathrm{h}} \lambda\right|_{\partial U}
$$

when evaluated in $\mathcal{E}_{L}, \mathfrak{F}_{U}$. In particular $\left.\mathcal{L}_{j V} \Omega_{L}\right|_{\partial U}=0$.

2. The Lie ideal of gauge variations with null boundary conditions

$$
\mathfrak{G}_{U} \subseteq \mathfrak{F}_{U}^{\mathrm{LH}}
$$

consists of those $X \in \hat{\mathfrak{G}}_{U}$ such that (8) holds together with

$$
\left.\mathrm{j} X\right|_{\partial U}=0 .
$$

Which means that there is no gauge action in the boundary.

The following assertions are used in the definition.

Lemma 5. The following inclusions are Lie ideal inclusions into Lie algebras:

$$
\mathfrak{G}_{U} \subseteq \mathfrak{F}_{U}^{\mathrm{LH}}, \mathfrak{G}_{U} \subseteq \hat{\mathfrak{F}}_{U}^{\mathrm{LH}}, \mathfrak{G}_{U} \subseteq \hat{\mathfrak{G}}_{U}, \mathfrak{F}_{U}^{\mathrm{LH}} \cap \hat{\mathfrak{G}}_{U} \subseteq \mathfrak{F}_{U}^{\mathrm{LH}}
$$

Proof. $X, X^{\prime} \in \mathfrak{G}_{U}$ imply that $\left.\mathrm{j}\left[X, X^{\prime}\right]\right|_{\partial U}=0$ hence $\mathfrak{G}_{U}$ is indeed a Lie algebra. To see that it is an ideal in $\hat{\mathfrak{F}}_{U}^{\mathrm{LH}}$ we just consider the fact that $\left.\mathrm{j}[X, V]\right|_{\partial U}=0$ for every $V \in \mathfrak{F}_{U}^{\mathrm{LH}}$. 
To see that $\mathfrak{G}_{U}$ is an ideal in $\mathfrak{F}_{U}^{\mathrm{LH}}$, derive vertically (8) and notice that $\sigma^{X}=-\mathrm{d}_{\mathrm{v}} \rho^{X}$ is null along $\partial U$ thanks to Lemma 4 , in particular $\left.\sigma^{X}\right|_{\partial U}$ is $\mathrm{d}_{\mathrm{h}}$-exact.

We claim that $\mathfrak{G}_{U}$ is an ideal of $\hat{\mathfrak{G}}_{U}$. For if $X \in \mathfrak{G}_{U}, X^{\prime} \in \mathfrak{G}_{U}$ then $\mathrm{j}\left[X, X^{\prime}\right]=\left.\left[\mathrm{j} X, \mathrm{j} X^{\prime}\right]\right|_{\partial U}$ vanishes.

Finally, to see that $\mathfrak{F}_{U}^{\mathrm{LH}} \cap \hat{\mathfrak{G}}_{U} \subseteq \mathfrak{F}_{U}^{\mathrm{LH}}$ is an ideal, $\mathrm{d}_{\mathrm{v}} \mathrm{l}_{\mathrm{j}[X, V]} \Omega_{L}$ is $\mathrm{d}_{\mathrm{h}}$-exact by (9).

\section{Linear Theory}

Recall that each fiber of $\pi: Y \rightarrow M$ is an affine bundle modeled over a linear bundle $\pi^{\mathrm{L}}: Y^{\mathrm{L}} \rightarrow M$ with $Y^{\mathrm{L}} \subseteq \Omega^{1}(M)$.

Since the space of YM solutions $\mathcal{A}_{U}$ is an affine space, take a fixed connection $\eta_{0} \in \mathcal{A}_{U}$, then $\varphi=\eta-\eta_{0} \in \Gamma\left(\left.Y^{\mathrm{L}}\right|_{U}\right)$ is such that $d \star d \varphi=0$. Here $\star$ denotes the Hodge star operator. In addition, there exists $V_{\varphi} \in \mathfrak{F}_{U}$, such that

$$
\mathrm{j} V_{\varphi}=\mathrm{j}\left(\sum_{i=1}^{n} \varphi^{i}(x) \frac{\partial}{\partial A^{i}}\right)
$$

Even though Equation (5) imposes a condition on-shell, i.e., on $\mathcal{E}_{L}$ for $V \in \mathfrak{F}_{U}$, the linearized equations, $d \star d \varphi=0$, induce $V_{\varphi} \in \mathfrak{F}_{U}$ that satisfies (5) off-shell, that is in $J Y$.

As a complementary definition to (10) we may define for every solution, $\eta \in \mathcal{A}_{U}$, and every first variation of solutions, $V \in \mathfrak{F}_{U}$ the section

$$
\eta_{V}=\mathrm{j} \eta^{*} V \in \Gamma\left(\left.Y^{\mathrm{L}}\right|_{U}\right) \subseteq \Omega^{1}(U) .
$$

Here we use the isomorphism, depending on a fixed connection, $\eta_{0} \in \mathcal{A}_{U}$, between the pullback $\mathrm{j} \eta_{0}^{*}\left(Y^{v}\right)$ of the vertical bundle $\pi^{v}:\left.Y^{v} \rightarrow J Y\right|_{U}$, and the linear bundle $\left.\pi^{\mathrm{L}}\right|_{U}: Y^{\mathrm{L}} \rightarrow U$.

For the previous definitions the following properties hold

$$
\mathrm{j} \eta^{*} V_{\eta_{V}}=\mathrm{j} \eta^{*} V, \quad \eta_{\left(V_{\varphi}\right)}=\varphi .
$$

The following assertion holds as an observation that will follow from Lemma 9.

Lemma 6. We have that $V_{\varphi} \in \mathfrak{F}_{U}^{\mathrm{LH}}$ for every $\varphi \in \Omega^{1}(U)$, solution of the linearized equation $d \star d \varphi=0$. Hence, $V_{\eta_{V}} \in \mathfrak{F}_{U}^{\mathrm{LH}}$ for every $V \in \mathfrak{F}_{U}$.

The following assertion holds for linear theories.

Lemma 7. For every solution, $\eta \in \mathcal{A}_{U}$, and every first variation of solutions, $V \in \mathfrak{F}_{U}$, in a linear theory, there exists $\varphi \in \Gamma\left(\left.Y^{\mathrm{L}}\right|_{U}\right)$ such that $\left.V\right|_{\mathrm{j} \eta(U)}=\left.V_{\varphi}\right|_{\mathrm{j} \eta(U)}$ or equivalently $\eta_{V}=\varphi$.

If we want to consider the gauge classes on $\mathcal{A}_{U}$ we can consider the gauge representatives consisting of Lorentz gauge fixing conditions, i.e., for every $\eta=\eta_{0}+\varphi \in \mathcal{A}_{U}$ there exists a gauge related

$$
\tilde{\eta}=\eta_{0}+\tilde{\varphi} \in \mathcal{A}_{U}, \quad d \star \tilde{\varphi}=0
$$

where $\tilde{\eta}-\eta=\tilde{\varphi}-\varphi \in \mathcal{G}_{U}$ being a gauge translation by exact 1-forms in $\mathcal{A}_{U}$.

Recall the Hodge-Morrey-Friedrichs $L^{2}$-ortogonal decomposition, see [19]. For null normal components we have,

$$
\Omega^{1}(U)=d \Omega_{D}^{0}(U) \oplus \mathfrak{H}_{N}^{1}(U) \oplus\left(\mathfrak{H}^{1}(U) \cap d \Omega^{0}(U)\right) \oplus d^{\star} \Omega_{N}^{2}(U)
$$

where

$$
\begin{aligned}
& \Omega_{N}^{1}(U):=\left\{\beta: \beta \in \Omega^{1}(U): i_{\partial U}^{*}(\star \beta)=0\right\} \\
& \mathfrak{H}_{N}^{1}(U):=\mathfrak{H}^{1}(U) \cap \Omega_{N}^{k}(U) .
\end{aligned}
$$


Given a fixed point, $\eta_{0} \in \mathcal{A}_{U}$, the linear space of Lorentz gauge fixing, $d \star \varphi=0$, defines a linear subspace

$$
\mathcal{L}_{U} \subseteq \mathfrak{H}_{N}^{1}(U) \oplus d^{\star} \Omega_{N}^{2}(U)
$$

of linearized solutions, $d^{\star} d \varphi=0$, such that there is a covering, $\mathfrak{e}_{\eta_{0}}(\varphi)=\left[\eta_{0}+\varphi\right]$,

$$
\mathfrak{e}_{\eta_{0}}: \mathcal{L}_{U} \rightarrow \mathcal{A}_{U} / \mathcal{G}_{U}
$$

of the $\left[\eta_{0}\right]$-componentspace of solutions modulo gauge, $\mathcal{A}_{U} / \mathcal{G}_{U}$.

The following results of this section recover the usual characterizations of gauge symmetries in $\mathfrak{G}_{U}$ as translations by exact forms.

Lemma 8. For every $X \in \hat{\mathfrak{G}}_{U}$ and $\eta \in \mathcal{A}_{U}, d \eta_{X}=0$.

Proof. If we calculate the square of the $L_{2}$-norm, $\left\|d \eta_{X}\right\|_{2}^{2}=\int_{U} d \eta_{X} \wedge \star d \eta_{X}$, of $d \eta_{X}$ where $\star$ stands for the Hodge star operator for the Riemannian metric $g$, then we get

$$
\int_{U} \eta_{X} \wedge \star d^{\star} d \eta_{X}-\int_{\partial U} \eta_{X} \wedge \star d \eta_{X}
$$

If $X \in \mathfrak{F}_{U}, d^{\star} d \eta_{X}=0$ then due to Lemma 4 , the norm $\left\|d \eta_{X}\right\|_{2}^{2}$ can be calculated as

$$
-\int_{\partial U} \eta_{X} \wedge \star d \eta_{X}=-\int_{\partial U} \mathrm{j} \eta^{*}\left(\rho^{X}\right) \wedge d \eta_{X}=\int_{\partial U} d\left(\mathrm{j} \eta^{*}\left(\rho^{X}\right)\right) \wedge \eta_{X}
$$

Recall (8) and that $\mathrm{j} \eta^{*}\left(\mathrm{~d}_{\mathrm{h}} \rho^{X}\right)=\mathrm{j} \eta^{*}\left(\iota_{\mathrm{j} X} \Omega_{L}\right)$. Hence

$$
d\left(\mathrm{j} \eta^{*}\left(\rho^{X}\right)\right) \wedge \eta_{X}=\mathrm{j} \eta^{*}\left(\iota_{\mathrm{j} X} \iota_{\mathrm{j} X} \Omega_{L}\right)=0
$$

Therefore $d \eta_{X}=0$.

Proposition 2. For every solution, $\eta \in \mathcal{A}_{U}$, and every gauge first variation with null boundary condition, $X \in \mathfrak{G}_{U}$, the induced 1 -form in the base, $\eta_{X}$, defined as in (11), is exact. Therefore, $\eta_{X} \in \mathcal{G}_{U}$.

Proof. We solve the Poisson BVP for $\psi: U \rightarrow \mathbb{R}$ with Dirichlet boundary conditions

$$
\left\{\begin{aligned}
\Delta \psi & =d^{\star} \eta_{X}, & & \text { in } U, \\
\left.\psi\right|_{\partial U} & =0, & & \text { in } \partial U .
\end{aligned}\right.
$$

Notice that the necessary integral condition for the Poisson equation $\int_{U} d \star \eta_{X} d v=0$ follows from the boundary condition $\left.\eta_{X}\right|_{\partial U}=0$.

Thus $\tilde{\eta}_{X}=\eta_{X}-d \psi$ is a solution of $d^{\star} d \tilde{\eta}_{X}=0$ with Lorentz gauge fixing condition $d^{\star} \tilde{\eta}_{X}=0$ and Dirichlet boundary condition.

Recall (13). Since $\eta_{X} \in \hat{\mathfrak{G}}_{U}$, according to Lemma $8, d \eta_{X}=0$ and $d \tilde{\eta}_{X}=0$.

There are two cases:

Case 1. The normal component $\partial \psi /\left.\partial x^{n}\right|_{\partial U}$ does not vanish. Here in local coordinates, $\partial U=$ $\left\{x^{n}=0\right\}$. Then $\tilde{\eta}_{X}$ is harmonic $\left(d \tilde{\eta}_{X}=0\right.$ and $\left.d^{\star} \tilde{\eta}_{X}=0\right)$. Therefore, it belongs to $\mathfrak{H}^{1}(U) \cap d \Omega^{0}(U)$, i.e., it is exact.

Case 2. $\partial \psi /\left.\partial x^{n}\right|_{\partial U}=0$, that is, $\tilde{\eta}_{X} \in \Omega_{N}^{1}(U)$. Then $\tilde{\eta}_{X} \in \mathfrak{H}_{N}^{1}(U) \cap \mathfrak{H}_{D}^{1}(U)$, i.e., $\tilde{\eta}_{X}=0$, where

$$
\begin{aligned}
& \Omega_{D}^{1}(U):=\left\{\beta: \beta \in \Omega^{1}(U): \beta(\xi)=0, \xi \in \mathfrak{X}(\partial U)\right\}, \\
& \mathfrak{H}_{D}^{1}(U):=\mathfrak{H}^{1}(U) \cap \Omega_{D}^{k}(U) .
\end{aligned}
$$

In any case $\tilde{\eta}_{X}$ is exact and so is $\eta_{X}$. 
Proposition 3. Take any solution $\eta$, and any gauge symmetry, $X \in \mathfrak{F}_{U}^{\mathrm{LH}} \cap \hat{\mathfrak{G}}_{U}$. Then there exists $X^{\prime} \in \hat{\mathfrak{G}}_{U}$ such that $X-X^{\prime} \in \mathfrak{G}_{U}$. Hence $\eta_{X-X^{\prime}} \in \mathcal{G}_{U}$ is exact.

Proof. Take $X \in \mathfrak{F}_{U}^{\mathrm{LH}} \cap \hat{\mathfrak{G}}_{U}$. According to the argument given in Proposition 2 we just need to show that the pullback $i_{\partial U}^{*} \eta_{X} \in \Omega^{1}(\partial M)$ is null for the inclusion $i_{\partial U}: \partial U \rightarrow U$. Then $\eta_{X}-d \psi$ would have null Dirichlet condition and would be exact for suitable $\psi$.

Notice that the following boundary conditions are in general different objects:

$$
i_{\partial U}^{*} \eta_{X},\left.\quad \eta_{X}\right|_{\partial U},\left.\quad(\mathrm{j} X)\right|_{\partial U},\left.\quad \mathrm{j}\right|_{\partial U}\left(\left.X\right|_{\partial U}\right) .
$$

Since $X \in \mathfrak{F}_{U}^{L H}$, then we are assuming a boundary condition on $X$, namely $\left.d_{v} \rho\right|_{\partial U}=\lambda$, with $d_{h} \lambda=$ 0 , when evaluating in $\mathcal{E}_{L}, \mathfrak{F}_{U}$. Due to Lemma 4 we have that $\left.X\right|_{\partial U}$ does not depend on vertical coordinates, $u^{j}$ when evaluating in $\mathcal{E}_{L}$.

We claim that indeed $i_{\partial u}^{*} \eta_{X}=0$. Recall that, according to Lemma 4 , for every $W \in \mathfrak{F} u$ we have

$$
\begin{gathered}
\int_{\partial U} \mathrm{j} \eta^{*}\left(\sum_{j=1}^{n-1} \omega^{n j} X^{j}\left(\frac{\mathrm{d} W^{j}}{\mathrm{~d} x^{n}}-\frac{\mathrm{d} W^{n}}{\mathrm{~d} x^{j}}\right) v^{n}\right)= \\
\int_{\partial U} \mathrm{j} \eta^{*}\left(\iota_{\mathrm{j} W} \iota_{\mathrm{j} X} \Omega_{L}\right)=\int_{\partial U} \mathrm{j} \eta^{*}\left(\iota_{\mathrm{j} W} \mathrm{~d}_{\mathrm{h}} \rho\right)=\int_{\partial U} d\left(\mathrm{j} \eta^{*}\left(\iota_{\mathrm{j} W} \rho\right)\right)=0 .
\end{gathered}
$$

Therefore, $\left.X^{j}(\mathrm{j} \eta)\right|_{\partial u}=0$ for $j=1, \ldots, n-1$, hence null Dirichlet boundary conditions hold for $\eta_{X}$. There exists a smooth function $f: U \rightarrow \mathbb{R}$ such that $i_{\partial U}^{*} d f=i_{\partial U}^{*} \eta_{X}=0$, and $\left.\frac{\partial f}{\partial x^{n}}\right|_{\partial U}=X^{n}(\mathrm{j} \eta)$. If

$$
\mathrm{j} X_{d f}=\mathrm{j}\left(\sum_{i=1}^{n} \frac{\partial f}{\partial x^{i}} \frac{\partial}{\partial u^{i}}\right),
$$

then $X_{\eta}^{\prime}:=\mathrm{j} \eta^{*}\left(X-X_{d f}\right)=\eta_{X}-d f$ has null both Neumann and Dirichlet conditions on $\partial U$. We just need to refine the choice of $f$, so that $\left.\mathrm{j} X^{\prime}\right|_{\partial U}=0$. Hence $X^{\prime} \in \mathfrak{G}_{U}$.

Theorem 1. There is an inclusion of the gauge quotients of Lie algebras,

$$
\mathfrak{F}_{U}^{\mathrm{LH}} / \mathfrak{G}_{U} \stackrel{\hat{\longrightarrow}}{\longrightarrow} \hat{\mathfrak{F}}_{U}^{\mathrm{LH}} / \hat{\mathfrak{G}}_{U}
$$

Proof. By the Second Isomorphism Theorem for Lie algebras

$$
\hat{\mathfrak{F}}_{U}^{\mathrm{LH}} / \hat{\mathfrak{G}}_{U} \simeq\left(\hat{\mathfrak{F}}_{U}^{\mathrm{LH}} / \mathfrak{G}_{U}\right) /\left(\hat{\mathfrak{G}}_{U} / \mathfrak{G}_{U}\right)
$$

Notice that

$$
\mathfrak{G}_{U} \subseteq \mathfrak{F}_{U}^{\mathrm{LH}} \cap \hat{\mathfrak{G}}_{U} \subseteq \operatorname{ker} \Psi
$$

where $\Psi$ is the Lie algebra morphism defined as the composition in the diagram below.

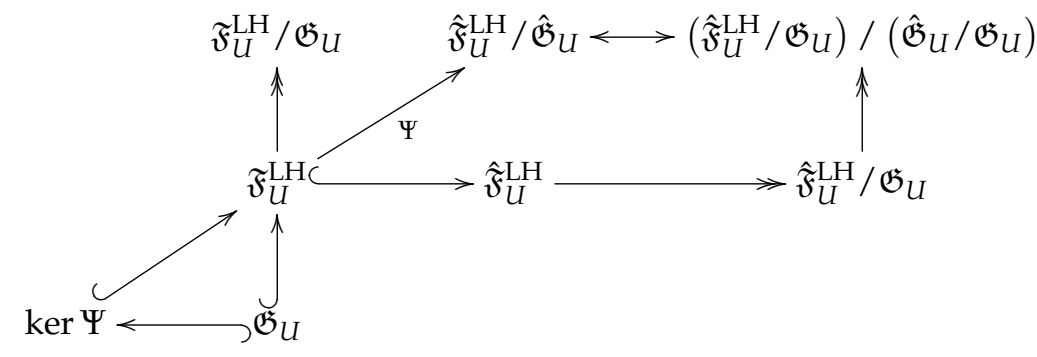


By the first isomorphism theorem, there exists an induced monomorphism $\tilde{\Psi}$ and a commutative diagram

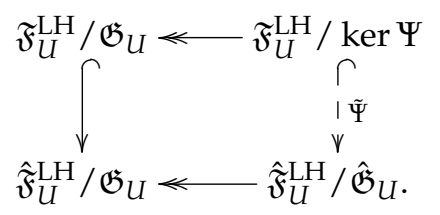

There is an inclusion $\mathfrak{F}_{U}^{\mathrm{LH}} \cap \hat{\mathfrak{G}}_{U} \subseteq \mathfrak{F}_{U}^{\mathrm{LH}}$. Hence ker $\Psi=\mathfrak{F}_{U}^{\mathrm{LH}} \cap \hat{\mathfrak{G}}_{U}$. By Proposition 3, the inclusion $\mathfrak{G}_{U} \subseteq \mathfrak{F}_{U}^{\mathrm{LH}} \cap \hat{\mathfrak{G}}_{U}$, is a section of the projection $\mathfrak{F}_{U}^{\mathrm{LH}} \cap \hat{\mathfrak{G}}_{U}-\rightarrow \mathfrak{G}_{U}$, given by $X \mapsto X-X^{\prime}$.

Therefore, we have the required inclusion

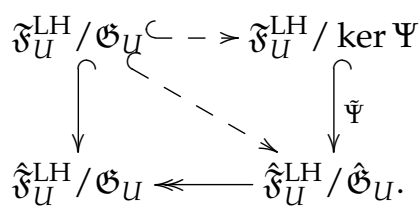

Recall that $H_{\mathrm{dR}}^{1}(U, \partial U) \simeq \mathfrak{H}_{D}^{1}(U)$ in the exact sequence,

$$
H_{\mathrm{dR}}^{1}(U, \partial U) \longrightarrow H_{\mathrm{dR}}^{1}(U) \stackrel{i_{\partial U}^{*}}{\longrightarrow} H_{\mathrm{dR}}^{1}(\partial U) .
$$

Hence, the demand in the proof of Proposition 3 for $i_{\partial u}^{*} \eta_{X}$ to be null is equivalent to demanding $\eta_{X}$ to lie into $\Omega_{D}^{1}(U)$. Thus, $\eta_{X}$ defines a relative cohomology class $\left[\eta_{X}\right] \in H_{\mathrm{dR}}^{1}(U, \partial U)$. Further considerations actually explain that $\left[\eta_{X}\right]=0$.

Proposition 4. If $H_{\mathrm{dR}}^{1}(U, \partial U)=0$, then $\mathfrak{F}_{U}^{\mathrm{LH}} / \mathfrak{G}_{U} \simeq \hat{\mathfrak{F}}_{U}^{\mathrm{LH}} / \hat{\mathfrak{G}}_{U}$.

Proof. For every $V \in \mathfrak{F}_{U}^{L H}$ we have that $\left.\mathrm{d}_{v} \iota_{\mathrm{j} V} \Omega_{L}\right|_{\partial U}=\mathrm{d}_{\mathrm{h}} \sigma^{V}$, with $\left.\sigma^{V}\right|_{\partial U}=\left.\mathrm{d}_{\mathrm{h}} \lambda\right|_{\partial U}$. Take $\eta \in \mathcal{A}_{U}$ any YM solution. For $\left.\eta_{V}\right|_{\partial U}=\left.\mathrm{j} \eta^{*} V\right|_{\partial U}$, we solve the Poisson BVP

$$
\begin{cases}\Delta \psi=d^{\star} \eta_{V}, & \text { in } U, \\ \partial \psi /\left.\partial x^{n}\right|_{\partial U}=-V^{n}(x), & \text { in } \partial U=\left\{x^{n}=0\right\},\end{cases}
$$

then $\eta_{V}$ may be gauge translated by an exact form $d \psi$ so that $\tilde{\eta}_{V}=\eta_{V}+d \psi$ has no normal components along $\partial U$ and satisfies $d \star \tilde{\eta}_{V}=0$ as well as the linearized YM equation, $d^{\star} d \tilde{\eta}_{V}=0$.

Notice that the induced linearized solution $X_{d \psi} \in \mathfrak{F}_{U}$ in fact belongs to $\hat{\mathfrak{E}}_{U} \cap \mathfrak{F}_{U}^{\mathrm{LH}}$.

By (13) $\tilde{\eta}_{V} \in \mathfrak{H}_{N}^{1}(U) \oplus\left(\mathfrak{H}^{1}(U) \cap d \Omega^{0}(U)\right) \oplus d^{\star} \Omega_{N}^{2}(U)$. For the coclosed projection $\eta_{V}^{\prime} \in \mathfrak{H}_{N}^{1}(U) \oplus$ $d^{\star} \Omega_{N}^{2}(U)$ of $\tilde{\eta}_{V}$, we have the orthogonal decomposition, $\eta_{V}^{\prime}=\eta_{V}^{\prime \prime} \oplus d^{\star} \chi$.

Consider the boundary conditions linear map, $\mathrm{d} r_{\partial U}[\eta]: \mathfrak{F}_{U}^{\mathrm{LH}} / \mathfrak{G}_{U} \rightarrow \mathcal{L}_{\partial U}$, such that

$$
\left(\mathrm{d} r_{\partial U}\right)[\eta](V)=\left(\eta_{V}^{\prime}\right)^{D} \oplus\left(\eta_{V}^{\prime}\right)^{N}:=\left[\iota_{\partial U}^{*} \eta_{V}^{\prime}\right] \oplus \star \partial u i_{\partial U}^{*}\left(\star d \eta_{V}^{\prime}\right),
$$

where the codomain is the linear space of Dirichlet-Neumann boundary conditions modulo gauge,

$$
\mathcal{L}_{\partial U}:=\left(\operatorname{ker} d^{\star \partial U} /\left(d \Omega^{0}(U) \cap \operatorname{ker} d^{\star} \partial u\right)\right) \oplus \operatorname{ker} d^{\star} \partial u .
$$

See [15] for further considerations of this space. Recall the isomorphisms

$$
\mathfrak{H}_{N}^{1}(U) \simeq H_{\mathrm{dR}}^{1}(U), \quad \mathfrak{H}_{D}^{1}(U) \simeq H_{\mathrm{dR}}^{1}(U, \partial U) .
$$


Since $\mathfrak{H}_{D}^{1}(U) \simeq H_{\mathrm{dR}}^{1}(U, \partial U)=0$, then by (16) we have $\mathfrak{H}_{N}^{1}(U) \subseteq H_{\mathrm{dR}}^{1}(\partial U)$. Hence, the closed projection of $i_{\partial U}^{*}\left(\eta_{V}^{\prime}\right) \in \Omega^{1}(\partial U)$ would have cohomology class $i_{\partial U}^{*}\left[\eta_{V}^{\prime \prime}\right]$ in $H_{\mathrm{dR}}^{1}(\partial U)$ induced by $\left[\eta_{V}^{\prime \prime}\right] \in$ $H_{\mathrm{dR}}^{1}(U)$. Therefore, $\left(\mathrm{d} r_{\partial U}\right)[\eta]$ is injective.

If we proceed as in the previous argument with $[V] \in \hat{\mathfrak{F}}^{\mathrm{LH}} / \hat{G}_{U}$, we can define an injective map $\widehat{\mathrm{d}} r_{\partial U}$ such that the following diagram commutes

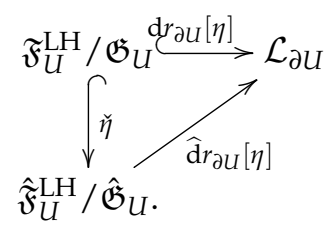

Notice that $\widehat{\mathrm{d}} r_{\partial U}[\eta]$ and $\mathrm{d} r_{\partial U}[\eta]$ have the same image.

Remark that we have the commutative diagram

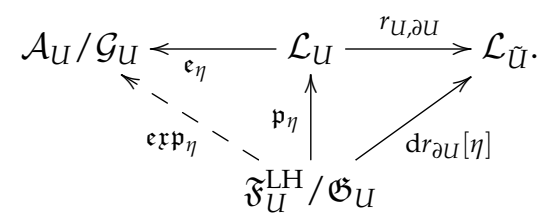

where

$$
\mathcal{L}_{\tilde{U}}:=r_{U, \partial U}\left(\mathcal{L}_{U}\right) \subseteq \mathcal{L}_{\partial U}
$$

with $r_{U, \partial U}$ the map of boundary conditions of solutions modulo gauge, see [15] for further properties of this map. Here we use axial gauge fixing in a tubular neighborhood of $\partial U$ as well as the linear map $r_{U, \partial U}(\varphi)=\varphi^{D} \oplus \varphi^{N}$ is defined in (17). The linear map $\mathfrak{p}$ is induced by $\mathfrak{p}_{\eta}(V)=\eta+\varphi$ where $\varphi \in \Omega^{1}(U)$ is a coclosed linearized solution, $d \star d \varphi=0$ such that $\eta_{V}=\varphi$, see notation (11).

By composing the projection $\mathfrak{p}_{\eta}$ with the map $\mathfrak{e}_{\eta}$ we get the map $\mathfrak{e x p}_{\eta}: \mathfrak{F}_{U}^{\mathrm{LH}} / \mathfrak{G}_{U} \rightarrow \mathcal{A}_{U} / \mathcal{G}_{U}$. Diagram (18) suggests that Hamiltonian first variation modulo gauge, $\mathfrak{F}_{U}^{\mathrm{LH}} / \mathfrak{G}_{U}$ is a Lie algebra isomorphic as linear space to the tangent space of the moduli space $\mathcal{A}_{U} / \mathcal{G}_{U}$ at $\eta$.

The following assertion related to Proposition 4 explains how the relative cohomology codifies the description of $\mathcal{A}_{U} / \mathcal{G}_{U}$ with respect to the boundary conditions, see also [15].

Proposition 5. $H_{\mathrm{dR}}^{1}(U, \partial U)=0$ if and only if $r_{\partial U}: \mathcal{L}_{U} \rightarrow \mathcal{L}_{\partial U}$ is injective and $r_{\partial U}: \mathcal{L}_{U} \rightarrow \mathcal{L}_{\tilde{U}}$ is a linear isomorphism.

\section{Poisson-Lie Algebra of Hamiltonian Observables}

Definition 3 (Hamiltonian observable currents). We say that an observable current $F \in \Omega^{n-1,0}\left(\left.\mathrm{JY}\right|_{U}\right)$ is a Hamiltonian observable current if there exist $V \in \mathfrak{F}_{U}$ and a residual form $\sigma^{F}$ such that the following relation holds when restricted to $\mathcal{E}_{L}$ and evaluated on $W \in \mathfrak{F}_{U}$,

$$
\left.\mathrm{d}_{\mathrm{v}} F\right|_{\mathcal{E}_{L}}=-\iota_{\mathrm{j} V} \Omega_{L}+\mathrm{d}_{\mathrm{h}} \sigma^{F}
$$

We denote the space of Hamiltonian observable currents over $U$ as $\widehat{\mathrm{HOC}}_{U}$. The evolutionary vector field $V$, is actually a locally Hamiltonian first variation, i.e., $V \in \hat{\mathfrak{F}}_{U}^{\mathrm{LH}}$. If in addition in (19) we have the boundary condition

$$
\left.\mathrm{d}_{\mathrm{v}} \sigma^{F}\right|_{\partial U}=\mathrm{d}_{\mathrm{h}} \lambda^{F}
$$

then we call F a Hamiltonian observable current with boundary condition. Here $V \in \mathfrak{F}_{U}^{\mathrm{LH}}$. We denote the space of these kind of observable currents as $\mathrm{HOC}_{U}$. 
Definition 4 (Helicity current). Suppose that $\varphi \in \Omega^{1}(U)$ is a solution of the linearized YM equation, $d \star d \varphi=0$. Define the $\varphi$-helicity current $a s$

$$
F^{\varphi}=\iota_{\mathrm{j} V_{\varphi}} \iota_{\mathrm{j} R} \Omega_{L} \in \Omega^{n-1,0}\left(\left.J Y\right|_{U}\right),
$$

where $R \in \mathfrak{F} U$ was defined in (6). More explicitly

$$
F^{\varphi}=\sum_{i=1}^{n}\left[\sum_{j=1}^{n} \omega^{i j}\left(\varphi^{j}(x) \cdot\left(A_{i}^{j}-A_{j}^{i}\right)-A^{j} \cdot\left(\frac{\partial \varphi^{j}(x)}{\partial x^{i}}-\frac{\partial \varphi^{i}(x)}{\partial x^{j}}\right)\right)\right] v_{i} .
$$

Form the very definition and the multysimplectic formula it can be seen that $\left.\mathrm{d}_{\mathrm{h}} \mathrm{F}^{\varphi}\right|_{\mathcal{E}_{L}}=0$.

Remark that we could have defined observable currents, $F^{\varphi}$, for any divergence-free $\varphi$ in $U$, $d \star \varphi=0$, with evolutionary Hamiltonian vector field, $V_{\varphi} \in \mathfrak{E v}\left(\left.J Y\right|_{U}\right)$, rather than in restricting ourselves to Hamiltonians first variations in $\mathfrak{F}_{U}$, just as the observables considered in [8]. Nevertheless, if we had adopted this definition, then we would have to restrict the domain of $F^{\varphi}$ and evaluate only ob solutions $\eta^{\prime}=\eta_{0}+\varphi^{\prime} \in \mathcal{A}_{U}$ with Lorentz gauge fixing (12), $\varphi^{\prime} \in \mathcal{L}_{U}$ in order to have local invariance $\left.\mathrm{d}_{\mathrm{h}} F^{\varphi}\right|_{\mathcal{E}_{L}}=0$.

From the following assertion it follows that helicity currents are Hamiltonian observable currents restricted to $U$, that is $F^{\varphi} \in \mathrm{HOC}_{U}$.

Lemma 9. The $\varphi$-helicity current, $F^{\varphi} \in \Omega^{n-1,0}\left(\left.\mathrm{~J} Y\right|_{U}\right.$, defines a locally Hamiltonian observable current with Hamiltonian $V_{\varphi} \in \mathfrak{F}_{U}^{\mathrm{LH}}$ whenever $d \star d \varphi=0$.

Proof. Recall the notation in (10). Notice that the relation $\mathrm{d}_{\mathrm{v}} F^{\varphi}+\iota_{\mathrm{j} V_{\varphi}} \Omega_{L}=0$ is valid off-shell. Therefore we have

$$
\left.\mathrm{d}_{\mathrm{v}} F^{\varphi}\right|_{\mathcal{E}_{L}}=-\iota_{\mathrm{j} V_{\varphi}} \Omega_{L}
$$

in particular when evaluated on $W \in \mathfrak{F}_{U}$.

Lemma 10. If $\varphi, \varphi^{\prime} \in \Omega^{1}(U)$ are solutions of $d \star d \varphi^{\prime}=0=d \star d \varphi$, then the Lie derivative, $\mathcal{L}_{\mathrm{j} V_{\phi^{\prime}}} F^{\varphi}$, lies in $\mathrm{HOC}_{U}$ with Hamiltonian $\left[V_{\varphi}, V_{\varphi^{\prime}}\right] \in \mathfrak{F}_{U}$. Under integration over $\Sigma$, it yields the symplectic product observable, associated to $\iota_{\mathrm{j}} V_{\varphi} \iota_{\mathrm{j}} V_{\varphi^{\prime}} \Omega_{L} \in \mathrm{HOC}_{U}$,

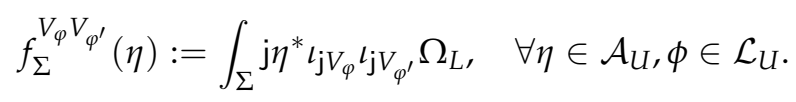

Proof. Notice that

$$
\mathcal{L}_{\mathrm{j} V_{\varphi^{\prime}}} F^{\varphi}=\iota_{\mathrm{j} V_{\varphi^{\prime}}} \mathrm{d}_{\mathrm{v}} F^{\varphi}=-\iota_{\mathrm{j} V_{\varphi^{\prime}}} \mathrm{j}_{\mathrm{j} V_{\varphi}} \Omega_{L}
$$

evaluated on $W \in \mathfrak{F}_{U}$ on Shell. On the other hand a general formula (9) states that

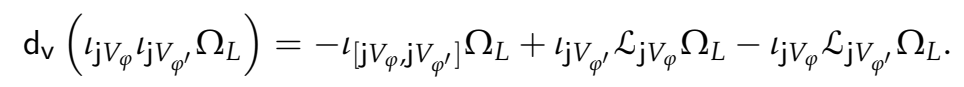

Therefore $\mathrm{d}_{\mathrm{v}}\left(\iota_{\mathrm{j} V_{\varphi}} \iota_{\mathrm{j} V_{\phi^{\prime}}} \Omega_{L}\right)=-\iota_{\left[\mathrm{j} V_{\varphi}, \mathrm{j} V_{\varphi^{\prime}}\right]} \Omega_{L}$. Recall that $\left[\mathrm{j} V_{\varphi,} \mathrm{j} V_{\varphi^{\prime}}\right]=\mathrm{j}\left[V_{\varphi^{\prime}}, V_{\varphi^{\prime}}\right]$, see for instance [20] por the explicit form of the Lie bracket of evolutionary vector fields. Hence $\left[V_{\varphi}, V_{\varphi^{\prime}}\right]$ is Hamiltonian first variation for $\iota_{\mathrm{j}} V_{\varphi} \iota_{\mathrm{j}} V_{\phi^{\prime}} \Omega_{L} \in \mathrm{HOC}_{U}$.

Define the family of $\varphi$-helicity observables as

$$
f_{\Sigma}^{\varphi}(\eta)=\int_{\Sigma}(\mathrm{j} \eta)^{*} F^{\varphi}, \quad \forall \eta \in \mathcal{A}_{U}
$$


We see that $f_{\Sigma}^{\varphi}$ is related to the anti-symmetric component of the helicity as bilinear form, see Section 1 , in the sense of (3). Notice also that $[\cdot, \cdot]_{\Sigma}$ is not necessarily symmetric, unless $\partial \Sigma=0$. Hence $f_{\Sigma}^{\varphi}$ not necessarily equals 0 .

We say that $f_{\Sigma}^{\varphi}$ is a Hamiltonian observable with Hamiltonian first variation $v_{\varphi}$ so that the following formal identity holds:

$$
\mathcal{L}_{w} f_{\Sigma}^{\varphi}(\eta)=-\omega_{\Sigma L}[\eta]\left(v_{\varphi}, w\right), \quad \forall w=\delta \eta, \forall \eta \in \mathcal{A}_{U} .
$$

Let us explain the formal notation of (21). Any first variation of solutions, $W \in \mathfrak{F}_{U}$, encodes a variation of any fixed solution $\eta \in \mathcal{A}_{U}$, which we denote as $w=\delta \eta$,

$$
w=\left.\frac{\mathrm{d} \phi^{\varepsilon}}{\mathrm{d} \varepsilon}\right|_{\varepsilon=0}, \quad \phi^{0}=\eta
$$

for a one-parameter family of smooth solutions $\phi^{\varepsilon} \in \mathcal{A}_{U}$. This means that $\left.\frac{\mathrm{d}\left(\mathrm{j} \eta^{\varepsilon}\right)}{\mathrm{d} \varepsilon}\right|_{\varepsilon=0}=\mathrm{j} W(\mathrm{j} \eta)$.

In the r.h.s. we have an evaluation of a symplectic form,

$$
\omega_{\Sigma L}[\eta](v, w):=\int_{\Sigma}(\mathrm{j} \eta)^{*} \Omega_{L}(\mathrm{j} V, \mathrm{j} W) .
$$

While in the 1.h.s. we have

$$
\mathcal{L}_{w} f_{\Sigma}^{\varphi}(\eta)=\left.\frac{d}{d \varepsilon}\right|_{\varepsilon=0} f_{\Sigma}^{\varphi}\left(\phi^{\varepsilon}\right),
$$

With this notation we suggest that we are modeling a Lie derivative $\mathcal{L}_{w}(\cdot)$ in the tangent space of the moduli space $\mathcal{A}_{U} / \mathcal{G}_{U}$, while $w=\delta \eta$ corresponds to local vector fields near $[\eta] \in \mathcal{A}_{U} / \mathcal{G}_{U}$.

If $X \in \mathfrak{G}_{U}$ corresponds to a first variation of a one-parametric family of gauge equivalent solutions, $\phi^{\varepsilon}$, then $\mathcal{L}_{x} f_{\Sigma}^{\varphi}=0$, which follows from $\left.\mathrm{jX}\right|_{\partial U}=0$. Thus $f_{\Sigma}^{\varphi}$ is well defined for the gauge class $\left[V_{\varphi}\right] \in \mathfrak{F}_{U}^{\mathrm{LH}} / \mathfrak{G}_{U}$.

Lemma 11. Consider the linear space

$$
\mathfrak{f}_{\Sigma U}:=\left\{f_{\Sigma}^{\varphi}: d \star d \varphi=0,\left[V_{\varphi}\right] \in \mathfrak{F}_{U}^{\mathrm{LH}} / \mathfrak{G}_{U}\right\} / \mathbb{R}
$$

where $f_{\Sigma}^{\varphi_{1}}-f_{\Sigma}^{\varphi_{2}}$ is a constant function iff represent the same $\mathbb{R}$-class. Then $f_{\Sigma} u$ is a Lie algebra with bracket

$$
\left\{\left[f_{\Sigma}^{\varphi}\right],\left[f_{\Sigma}^{\varphi^{\prime}}\right]\right\}_{\Sigma}=\left[f_{\Sigma}^{V_{\varphi} V_{\varphi^{\prime}}}\right]
$$

which means

$$
\left\{f_{\Sigma}^{\varphi}, f_{\Sigma}^{\varphi^{\prime}}\right\}_{\Sigma}=f_{\Sigma}^{V_{\varphi} V_{\varphi^{\prime}}}+\text { const. }
$$

Proof. Let $\varphi, \varphi^{\prime}$ be 1-forms as in the hypothesis. As in the proof of Lemma 10, recall that

$$
\mathrm{d}_{\mathrm{v}}\left(\iota_{\mathrm{j} V_{\varphi}} \iota_{\mathrm{j} V_{\varphi^{\prime}}} \Omega_{L}\right)=-\iota_{\mathrm{j}}\left[V_{\varphi}, V_{\varphi^{\prime}}\right] \Omega_{L} .
$$

There are gauge translations $X=X_{\psi}, X^{\prime}=X_{\psi^{\prime}} \in \mathfrak{G}_{U}, \psi, \psi^{\prime}: U \rightarrow \mathbb{R}$ such that the gauge translations $V, V^{\prime}$ are divergence-free, see for instance the Appendix [15]. Recall that $V, V^{\prime}$ are defined by $\varphi-d \psi, \varphi^{\prime}-d \psi^{\prime}$, respectively. Hence $V=V_{\varphi}-X$ and $V^{\prime}=V_{\varphi^{\prime}}-X^{\prime}$. By (9)

$$
-\iota_{\mathrm{j}}\left[V_{\varphi}, V_{\phi^{\prime}}\right] \Omega_{L}=-\iota_{\mathrm{j}\left[V, V^{\prime}\right]} \Omega_{L}-\iota_{\mathrm{j}\left[V, X^{\prime}\right]} \Omega_{L}-\iota_{\mathrm{j}\left[X, V^{\prime}\right]} \Omega_{L}-\iota_{\mathrm{j}\left[X, X^{\prime}\right]} \Omega_{L}
$$


Hence

$$
\mathrm{d}_{\mathrm{v}}\left(\iota_{\mathrm{j} V_{\varphi}} \iota_{\mathrm{j} V_{\phi^{\prime}}} \Omega_{L}\right)=-\iota_{\mathrm{j}\left[V, V^{\prime}\right]} \Omega_{L}+\mathrm{d}_{\mathrm{h}} \sigma^{V V^{\prime}}
$$

with

$$
\sigma^{V V^{\prime}}=-\rho^{\left[V, X^{\prime}\right]}-\rho^{\left[X, V^{\prime}\right]}-\rho^{\left[X, X^{\prime}\right]} .
$$

Denote $\tilde{\varphi} \in \Omega^{1}(U)$ as the a 1 -form such that $\left[V, V^{\prime}\right]=V_{\tilde{\varphi}}$. In local coordinates:

$$
\tilde{\varphi}^{j}=\sum_{i=1}^{n} \varphi_{1}^{i}(x) \frac{d \varphi_{2}^{j}(x)}{d x^{i}}-\varphi_{2}^{i}(x) \frac{d \varphi_{1}^{j}(x)}{d x^{i}}, \quad \varphi_{1}=\varphi-d \psi, \varphi_{2}=\varphi^{\prime}-d \psi^{\prime}, .
$$

Recall that divergence-free vector fields form a Lie algebra, that is $d \star \tilde{\varphi}=0$. Then

$$
\mathrm{d}_{\mathrm{v}}\left(F^{\tilde{\varphi}}+\iota_{\mathrm{j} V_{\varphi}} \iota_{\mathrm{j}} V_{\varphi^{\prime}} \Omega\right)=\mathrm{d}_{\mathrm{h}} \sigma^{V V^{\prime}} .
$$

Therefore,

$$
\mathcal{L}_{w}\left(f_{\Sigma}^{V_{\varphi} V_{\varphi^{\prime}}}-f_{\Sigma}^{\tilde{\varphi}}\right)=0,
$$

for every variation of solutions $w$ associated to every $W \in \mathfrak{F}_{U}$. See the explanation of the notation in (21). Hence $f_{\Sigma}^{V_{\varphi} V_{\varphi^{\prime}}}=f_{\Sigma}^{\tilde{\varphi}}+$ const.

We claim that $\mathfrak{f}_{\Sigma U}$ yields a family of local observables sufficiently rich to separate solutions, see also [7]. Suppose that we consider a non-gauge variation $v=\delta \eta$ of a solution $\eta \in \mathcal{A}_{U}$. More precisely, take a one-parametric family of solutions $\eta^{\varepsilon}=\eta+\varepsilon \varphi$ encoded by the symmetry $V \in \mathfrak{F}_{U}$, that is $\left.\frac{\mathrm{d}}{\mathrm{d} \varepsilon}\right|_{\varepsilon=0} \mathrm{j}\left(\eta^{\varepsilon}\right)=\mathrm{j} V(\mathrm{j} \eta)$. Without loss of generality we can also suppose that $V=V_{\varphi}$ with $d \star d \varphi=0$. Hence, for any $0 \neq[V] \in \mathfrak{F}_{U}^{\mathrm{LH}} / \mathfrak{G}_{U}$, there exists $[W] \in \mathfrak{F}_{U}^{\mathrm{LH}} / \mathfrak{G}_{U},[W] \neq 0$, such that $\mathrm{j}^{*}\left(\iota_{\mathrm{j} V} \iota_{\mathrm{j} W} \Omega_{L}\right)$ in a suitable open $n$-dimensional ball $U^{\prime} \subseteq U$. We choose an embedded $(n-1)$-dimensional ball, $\Sigma^{\prime} \subseteq U^{\prime}, \partial \Sigma^{\prime} \subseteq \partial U^{\prime}$ such that

$$
\int_{\Sigma^{\prime}} \mathrm{j} \eta^{*}\left(\iota_{\mathrm{j}} W_{\phi^{\prime}} \iota_{\mathrm{j} V_{\varphi}} \Omega_{L}\right) \neq 0
$$

for $W=W_{\varphi^{\prime}}$ associated to $\varphi^{\prime} \in \Omega^{1}(U), d \star d \varphi^{\prime}=0$, a non trivial solution to linearized equations in $U^{\prime}$ that also vanishes in the exterior of $U^{\prime}$.

We then extend $\Sigma^{\prime}$ to $\Sigma \subseteq U, \partial \Sigma \subseteq \partial U$, such that $f_{\Sigma}^{V W}(\eta) \neq 0$. The variation of $f_{\Sigma}^{\varphi}$ along $w$ in the space of YM solutions is

$$
\mathcal{L}_{w} f_{\Sigma}^{\varphi}(\eta)=f_{\Sigma}^{V W}(\eta) \neq 0
$$

Remark that for every YM solution $\eta \in \mathcal{A}_{U}$ and for every variation $V \in \mathfrak{F}_{U}$, if $\varphi=\mathrm{j} \eta^{*} V$, then and $\left.\mathrm{j} V_{\varphi}\right|_{\mathrm{j} \eta}=\left.\mathrm{j} V\right|_{\mathrm{j} \eta}$. Thus we could change notation and index the family $\left\{f_{\Sigma}^{\varphi}\right\}$ as $\left\{f_{\Sigma}^{V}\right\}$ where we take $V$ in $\mathfrak{F}_{U}^{\mathrm{LH}}$.

We summarize the results exposed in this section in the following result and regard the family of observables $\left\{f_{\Sigma}^{V}\right\}$ as a "Darboux local coordinate system" for our gauge field theory.

Theorem 2 (Darboux's Theorem). Given $\eta \in \mathcal{A}_{U}$ a fixed $Y M$ solution. For each $\Sigma \subseteq U$ an admissible hypersurface, $\partial \Sigma \subseteq \partial U$, with relative homology class $[\Sigma] \in H_{n-1}(U, \partial U)$, there exists an infinite dimensional gauge invariant Lie algebra (modulo constant functions)

$$
\mathfrak{f}_{\Sigma U}=\left\{f_{\Sigma}^{V}:[V] \in \mathfrak{F}_{U}^{\mathrm{LH}} / \mathfrak{G}_{U}\right\} / \mathbb{R}
$$

such that the following assertions hold:

1. $\mathfrak{f}_{\Sigma U}$ is gauge invariant: If $X$ is a variation of one-parametric family of gauge equivalent solutions then $\mathcal{L}_{x} f_{\Sigma}^{V}=0$. Moreover, $\left[f_{\Sigma}^{V}\right] \in \mathfrak{f}_{\Sigma U}$ depends just on the gauge $\mathfrak{G}_{U}$-class, $[V] \in \mathfrak{F}_{U}^{\mathrm{LH}} / \mathfrak{G}_{U}$. 
2. Each variation $V$ is in fact locally Hamiltonian, $V \in \mathfrak{F}_{U}^{\mathrm{LH}}$ hence $f_{\Sigma}^{V}$ is an observable that satisfies the Hamilton's equation (recall notation in (21)):

$$
\mathcal{L}_{w} f_{\Sigma}^{V}(\eta)=-\omega_{\Sigma L}[\eta](v, w), \quad \forall w=\delta \eta
$$

3. $\mathfrak{f}_{\Sigma} U$, locally separates solutions near $\eta$ : For every non-gauge variation $v=\delta \eta$ modeled by $V \in \mathfrak{F}_{U}$, there exists a locally Hamiltonian variation $w$ modeled by $W \in \mathfrak{F}_{U}^{\mathrm{LH}}$ and $\Sigma \subseteq U$ with

$$
\mathcal{L}_{v} f_{\Sigma}^{W}(\eta) \neq 0
$$

The following commutative diagram of Lie algebra morphisms and vertical exact sequences summarizes our results

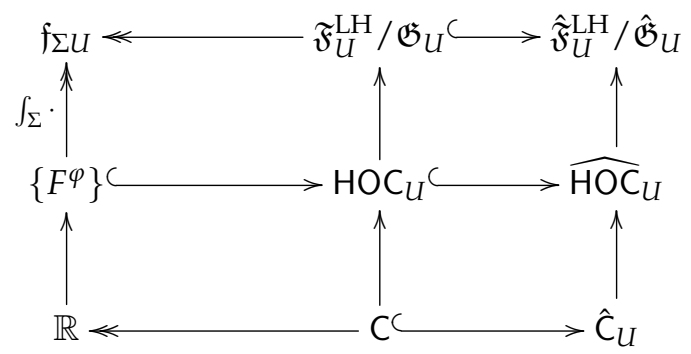

where $C_{U}$ denote subset of the the constant observable currents

$$
\hat{\mathrm{C}}_{U}:=\left\{F \in \widehat{\mathrm{HOC}}_{U}:\left.\left(\mathrm{d}_{\mathrm{v}} F-\mathrm{d}_{\mathrm{h}} \sigma^{F}\right)\right|_{\widetilde{F}_{U}, \mathcal{E}_{L}}=0\right\}
$$

with the additional boundary condition $\left.\left(\mathrm{d}_{\mathrm{v}} \sigma^{F}-\lambda^{F}\right)\right|_{\mathfrak{F}_{U}, \mathcal{E}_{L}}=0,\left.\mathrm{~d}_{\mathrm{h}} \lambda^{F}\right|_{\mathfrak{F}_{U}, \mathcal{E}_{L}}=0$.

Definition 5 (Poisson algebra). Let $\Sigma$ be any admissible hypersurface $\Sigma \subseteq U$. The (polynomial) Poisson algebra of helicity Hamiltonian observables,

$$
\left(\mathcal{P}\left(\mathfrak{f}_{\Sigma U}\right),\{\cdot, \cdot\}_{\Sigma}\right)
$$

is generated by the Lie algebra $\mathfrak{f}_{\Sigma U}=\left\{\left[f_{\Sigma}^{\varphi}\right]: \mathcal{A}_{U} / \mathcal{G}_{U} \rightarrow \mathbb{R}\right\}$.

The proof of the following assertion follows from the fact that the space of boundary conditions of solutions, $\mathcal{L}_{\tilde{U}} \subseteq \mathcal{L}_{\partial U}$, is a Lagrangian subspace with respect to the symplectic form $\omega_{\partial U, L}$, see [21].

Proposition 6. For a hypersurface $\Sigma \subseteq \partial U$ (such that $[\Sigma]=0 \in H_{n-1}(U, \partial U)$ ) and for its complement, $\Sigma^{\prime}=U-\Sigma \subseteq \partial U$, the corresponding observables uniquely define an observable

$$
f_{\partial \Sigma}^{\varphi}:=f_{\Sigma}^{\varphi}=-f_{\Sigma^{\prime}}^{\varphi} \in \mathfrak{f}_{\Sigma U}
$$

associated to the oriented and closed $(n-2)$-dimensional boundary $\partial \Sigma \subseteq \partial U$.

The Lie algebra

$$
\mathfrak{f}_{\partial U}:=\left\{f_{\partial \Sigma}^{\varphi}: \Sigma \subseteq \partial U\right\} / \mathbb{R}
$$

will suffice to separate boundary conditions of solutions, while the Lie algebras $\mathfrak{f}_{\Sigma U}$ corresponding to $0 \neq[\Sigma] \in H_{n-1}(U, \partial U)$ will be necessary if we want to separate solutions yielding the same boundary conditions, hence in the fibers of $r_{U, \partial U}: \mathcal{L}_{U} \rightarrow \mathcal{L}_{\tilde{U}} \subseteq \mathcal{L}_{\partial U}$. This happens when $H_{\mathrm{dR}}^{1}(U, \partial U) \neq 0$ according to Proposition 5. This also allows us to consider the fibers of $r_{U, \partial U}: \mathcal{L}_{U} \rightarrow \mathcal{L}_{\tilde{U}}$ as the 
symplectic leafs the coisotropic linear space $\mathcal{L}_{U}$. This image has been described in detail for the moduli space $\mathcal{A}_{U} / \mathcal{G}_{U}$ of non-abelian YM solutions in the two dimensional case, see for instance [22].

\section{Gluing Observable Currents}

Suppose that a region $U$ is obtained by gluing $U_{1}, U_{2}$ along the closed hypersurfaces $\Sigma_{1} \subseteq$ $\partial U_{1}, \Sigma_{2} \subseteq \partial U_{2}$, to avoid corners case we suppose $\partial \Sigma_{1}=\varnothing=\partial \Sigma_{2}$. This includes an isometry of $\Sigma_{1}$ with $\Sigma_{2}$ together with the compatibility of normal derivatives of the metric. We also suppose that the principal bundle $\mathcal{P}$ over $U$ is induced by the corresponding principal bundle $\mathcal{P}_{1}, \mathcal{P}_{2}$ over $U_{1}, U_{2}$. From the projection map $p: U_{1} \times U_{2} \rightarrow U$ we fix base points $\eta \in \mathcal{A}_{U}$ obtained by gluing $p^{*} \eta_{i} \in \mathcal{A}_{U_{i}}, i=1,2$.

Suppose that $V_{i} \in \mathfrak{F}_{U_{i}}, i=1,2$ satisfy the continuity gluing condition along $\Sigma_{i}$

$$
\mathrm{j}_{\Sigma}\left(\left.V_{1}\right|_{\Sigma_{1}}\right)=\mathrm{j}_{\Sigma}\left(\left.V_{2}\right|_{\Sigma_{2}}\right)
$$

and denote those couples $\left(V_{1}, V_{2}\right)$ satisfying (26) as $\mathfrak{F}_{U_{1}} \#_{\Sigma} \mathfrak{F}_{U_{2}}$, where $\Sigma=p\left(\Sigma_{i}\right) \subseteq U$. It is a Lie subalgebra of $\mathfrak{F}_{U_{1}} \oplus \mathfrak{F}_{U_{2}}$. The continuity gluing condition (26) is trivially satisfied for the gauge Lie algebras so that $\mathfrak{G}_{U_{1}} \#_{\Sigma} \mathfrak{G}_{U_{2}}=\mathfrak{G}_{U_{1}} \oplus \mathfrak{G}_{U_{2}}$, hence there is a well defined Lie algebra

$$
\mathfrak{F}_{U_{1}} \#_{\Sigma} \mathfrak{F}_{U_{2}} / \mathfrak{G}_{U_{1}} \#_{\Sigma} \mathfrak{G}_{U_{2}} \subseteq \mathfrak{F}_{U_{1}} \oplus \mathfrak{F}_{U_{2}} / \mathfrak{G}_{U_{1}} \#_{\Sigma} \mathfrak{G}_{U_{2}}
$$

Let $\hat{\mathfrak{G}}_{U_{1}}^{\Sigma} \subseteq \hat{\mathfrak{G}}_{U_{1}}$ denote those gauge variations whose jet vanish along the boundary components of $\partial U_{1}$ except for $\Sigma_{1}$. Similarly define $\hat{\mathfrak{G}}_{U_{2}}^{\Sigma}$. If we define

$$
\mathfrak{G}_{\Sigma}=\hat{\mathfrak{G}}_{U_{1}}^{\Sigma} \#_{\Sigma} \hat{\mathfrak{G}}_{U_{2}}^{\Sigma} /\left(\mathfrak{G}_{U_{1}} \#_{\Sigma} \mathfrak{G}_{U_{2}}\right)
$$

then by an Isomorphism Theorem for Lie algebras,

$$
\mathfrak{F}_{U_{1}} \#_{\Sigma} \mathfrak{F}_{U_{2}} / \hat{\mathfrak{G}}_{U_{1}}^{\Sigma} \#_{\Sigma} \hat{\mathfrak{G}}_{U_{2}}^{\Sigma} \simeq\left(\mathfrak{F}_{U_{1}} \#_{\Sigma} \mathfrak{F}_{U_{2}} / \mathfrak{G}_{U_{1}} \#_{\Sigma} \mathfrak{G}_{U_{2}}\right) / \mathfrak{G}_{\Sigma} .
$$

There is a commutative diagram of linear maps as follows. Recall the gluing procedure for abelian YM, see [15]. The doted arrow is a Lie algebra morphism.

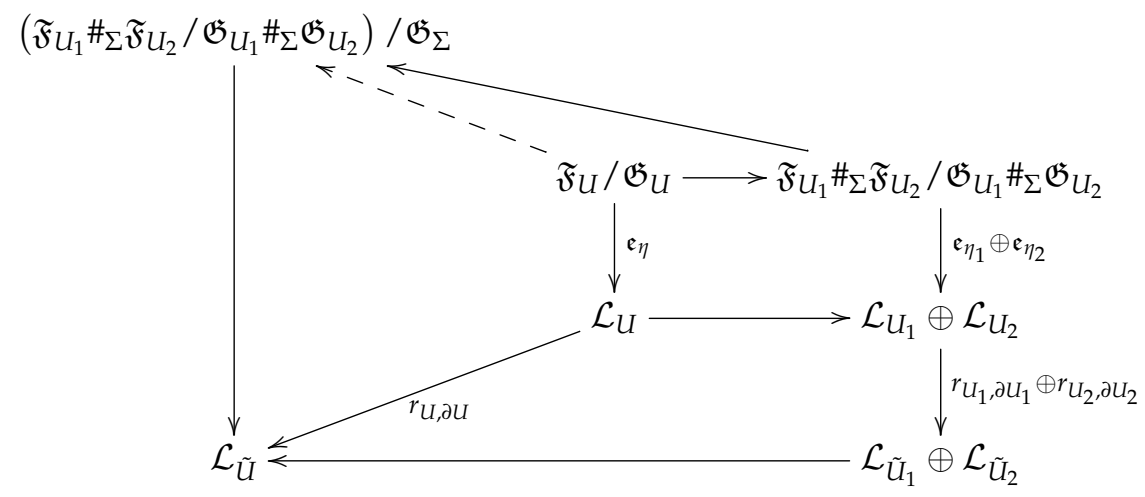

From the Lagrangian embedding of $\mathcal{L}_{\tilde{U}}$ with respect to the symplectic structure, $\omega_{\partial U, L}$, it follows that the Dirichlet conditions along $\Sigma_{1}$ and $\Sigma_{2}$ completely determine the Neumann conditions in $U_{1}$ and $U_{2}$, respectively. Here we consider an axial gauge fixing for solutions in $\partial U$ satisfying also the Lorentz gauge fixing condition in $\partial U$, see Appendix in [15]. This means that the continuous gluing condition (26) will suffice to reconstruct modulo gauge the first variation $V_{\varphi}=V_{\varphi_{1}} \# V_{\varphi_{2}}$ for $V_{\varphi_{i}} \in \mathfrak{F}_{U_{i}}, i=1,2$ disregarding the normal derivatives along $\Sigma$. This proves the following assertion 
Theorem 3 (Gluing of symmetries modulo gauge). There is an isomorpmhism of Lie algebras

$$
\left(\mathfrak{F}_{U_{1}} \#_{\Sigma} \mathfrak{F}_{U_{2}} / \mathfrak{G}_{U_{1}} \#_{\Sigma} \mathfrak{G}_{U_{2}}\right) / \mathfrak{G}_{\Sigma} \simeq \mathfrak{F}_{U} / \mathfrak{G}_{U}
$$

\section{Outlook: Further Problems}

We just remark that in further directions of research. In the first place, it is highly desirable to see whether or not $f_{\Sigma}^{V V^{\prime}}$ observables can be defined for non abelian (non-linear) YM equations and if it will suffice to separate solutions just as in Theorem 2. Extension of the variationa bicomplex ttreatment need to be extended to non-local first variations to get enough observables to separate solutions. The existence of a Jacobi bracket needs also to be verified in this case. Gluing properties for observables need also to be developed and explained in detail. Namely the continuous gluing of currents $\mathrm{HOC}_{U_{1}} \#_{\Sigma} \mathrm{HOC}_{U_{2}}$ in relation to $\mathrm{HOC}_{U}$, as well as the gluing $f_{\Sigma^{\prime}}^{V_{1}} \#_{\Sigma} f_{\Sigma^{\prime \prime}}^{V_{2}}$ for hypersurfaces $\Sigma^{\prime} \subseteq U_{1}, \Sigma^{\prime} \subseteq U^{\prime \prime}$ intersecting transversally the gluing boundary component $\Sigma_{i}, i=1,2$. Finally, considerations of Riemannian manifolds with corners may introduce further difficulties in the results we have established for the smooth boundary case.

Funding: This research was funded by CONACYT-MEXICO, grant number 58132.

Acknowledgments: The author thanks J. A. Zapata since most of the results of this article arise as a particular application of the results sketched in a joint work [23] see also [7]. Along the review of [23] many clarifications came from correspondence with Luca Vitagliano and notes from an anonymous referee who provided many clarifications for the difficulties arising in non-linear field theories.

Conflicts of Interest: The authors declare no conflict of interest.

\section{Abbreviations}

The following abbreviations are used in this manuscript:

$\begin{array}{ll}\text { PDE } & \text { PArtial differential equation } \\ \text { YM } & \text { Yang-Mills } \\ \text { BVP } & \text { Boundary value problem }\end{array}$

\section{Appendix A. Variational Bicomplex Formalism}

For the convenience of the reader, in this section we fix notation by recalling basic definitions of the variational formalism for variational PDEs taken from [3,11,18,20,24,25].

Let $M$ be an $n$-dimensional manifold, and let $\pi: Y \rightarrow M$ be a fiber bundle with $m$-dimensional fiber $\mathcal{F}$. Denote its sections or histories as $\Gamma\left(\left.Y\right|_{U}\right)$ where $U \subseteq M$ is a compact domain with piecewise smooth boundary.

The $k$-jet bundle $\pi_{k, 0}: \mathrm{J}^{k} Y \rightarrow Y, k=1,2, \ldots$ On $\pi^{-1}(U) \subset Y$ take the local coordinates

$$
\left(x ; u^{(k)}\right):=\left(x_{1}, \ldots, x_{i}, \ldots, x_{n} ; u^{1}, \ldots, u^{a}, \ldots, u^{m} ; \ldots, u_{I}^{a}, \ldots\right) \in \mathrm{J}^{k} \pi^{-1}(U)
$$

where $i=1, \ldots, n ; a=1, \ldots, m$; and $I=\left(i_{1}, \ldots, i_{n}\right)$ denotes a multiindex of degree $|I|:=i_{1}+\cdots+i_{n}=$ $0,1, \ldots, k, i_{j} \geq 0, i_{j} \in \mathbb{Z}$. For $I=\varnothing$, we define $u_{\varnothing}^{a}=u^{a}$. We denote the projection of the $(k+1)$-jet onto the $k$-jet as $\pi_{k+1, k}: \mathrm{J}^{k+1} Y \rightarrow \mathrm{J}^{k} Y$. For a section $\phi: M \rightarrow Y$, we denote its $k$-jet as $\mathrm{j}^{k} \phi: M \rightarrow \mathrm{J}^{k} Y$, where

$$
\mathrm{j}^{k} \phi(x)=\left(\phi^{1}(x), \ldots, \phi^{m}(x) ; \ldots, \frac{\partial^{|I|} \phi^{a}}{\partial x_{1}^{i_{1}} \ldots \partial x_{n}^{i_{n}}}, \ldots\right)
$$

Denote the space of $p$-forms on $J^{k} Y$ as $\Omega^{p}\left(J^{k} Y\right)$. For the decomposition $p=r+s$, denote the space of $r$-horizontal and $s$-vertical forms on $J^{k} Y$ as $\Omega^{r, s}\left(J^{k} Y\right)$, have as basis the $(r+s)$-forms $\vartheta_{I_{1}}^{a_{1}} \wedge \cdots \wedge \vartheta_{I_{s}}^{a_{s}} \wedge d x_{j_{1}} \wedge \cdots \wedge d x_{j_{r}}$, where 
The Cartan distribution on $J^{k} Y$ is generated by the basis of contact 1-forms (A1)

$$
\vartheta_{I}^{a}:=d u_{I}^{a}-\sum_{j=1}^{n} u_{(I, j)}^{a} d x_{j} \in \Omega^{1}\left(J^{|I|+1} Y\right),|I| \leq k-1, a=1, \ldots, m
$$

The vertical differential $d_{v}$ for $F \in \Omega^{0}\left(J^{k} Y\right)$ defined as

$$
\mathrm{d}_{\mathrm{v}}: \Omega^{r, s}\left(\mathrm{~J}^{k} Y\right) \rightarrow \Omega^{r, s+1}\left(\mathrm{~J}^{k} Y\right), \quad \mathrm{d}_{\mathrm{v}} F:=\sum_{0 \leq|I| \leq k} \sum_{a=1}^{m} \frac{\partial F}{\partial u_{I}^{a}} \vartheta_{I}^{a},
$$

then we are forced to consider the horizontal differential with range in the $(p+1)$-forms in $\mathrm{J}^{k+1} Y$

$$
\mathrm{d}_{\mathrm{h}}: \Omega^{r, s}\left(\mathrm{~J}^{k} Y\right) \rightarrow \Omega^{r+1, s}\left(\mathrm{~J}^{k+1} Y\right), \quad \mathrm{d}_{\mathrm{h}} F:=\sum_{i=1}^{n}{\frac{\mathrm{d}}{\mathrm{d} x_{i}}}^{(k+1)}(F) d x_{i} .
$$

where

$$
\left(\frac{\mathrm{d}}{\mathrm{d} x_{i}}\right)^{(k)}:=\frac{\partial}{\partial x_{i}}+\sum_{0 \leq|J| \leq k-1} \sum_{a=1}^{m} u_{(J, i)}^{a} \frac{\partial}{\partial u_{J}^{a}} .
$$

The injective limit $\Omega^{r, s}(J Y):=\lim _{\pi_{k+1, k}^{*}} \Omega^{r, s}\left(\mathrm{~J}^{k} Y\right)$, models the $p$ forms in the infinite jet space $J Y=\lim _{\pi_{k+1, k}} \mathrm{~J}^{k} Y$. We have the identities

$$
d_{v}^{2}=0, \quad d_{h}^{2}=0, \quad d_{v} d_{h}+d_{h} d_{v}=0
$$

Hence, the following diagram commutes

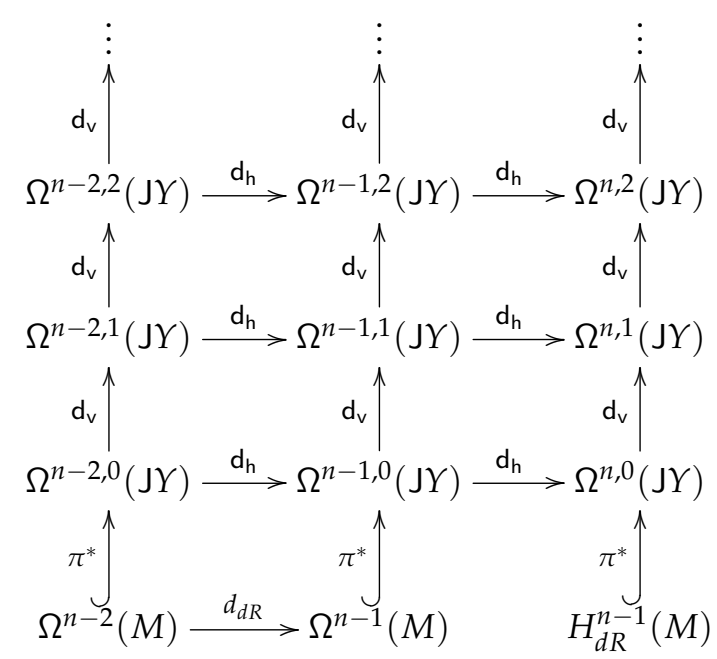

Derivations in the algebra of smooth functions on $\Omega^{0}(J Y)$,

$$
V=\sum_{i=1}^{n} a_{i}(x ; u) \frac{\partial}{\partial x_{i}}+\sum_{a=1}^{m} V^{a}(x ; u) \frac{\partial}{\partial u^{a}}
$$


are in correspondence with sections $V \in \Gamma\left(\pi_{\infty, 0}^{*}\left(\pi^{\vee}\right)\right)$, where $\pi_{\infty, 0}^{*}\left(\pi^{\vee}\right)$ is the pullback under $\pi_{\infty, 0}$ : $J Y \rightarrow Y$ of the vertical (vector) bundle, $\pi^{\vee}: Y^{\vee} \rightarrow Y$, whose fiber at each $(x, u) \in Y$ is consists of the vertical fibers $Y_{(x, u)}^{v}=T_{(x, u)} \pi^{-1}(x)$. In fact, its prolongations

$$
\mathrm{j}^{k} V=\sum_{i=1}^{n} a_{i}(x ; u)\left(\frac{\mathrm{d}}{\mathrm{d} x_{i}}\right)^{(k)}+\sum_{0 \leq|I| \leq k-1} \sum_{a=1}^{m} b_{I}^{a}\left(x ; u^{(k)}\right) \frac{\partial}{\partial u_{I}^{a}}
$$

where $b_{I}^{a}:=D_{I}^{(k)} V^{a}+\sum_{j=1}^{n} u_{j I}^{a} a_{j},|I| \leq k-1$, act as infinitesimal symmetries of the Cartan distribution in $J Y$ in the sense that

$$
\mathcal{L}_{\mathrm{j} V} \vartheta_{I}^{a}=0 .
$$

Here the horizontal derivative operator $D_{I}^{(k)}$ equals $D_{i_{1}}^{(k)} \circ D_{i_{2}}^{(k)} \circ \cdots \circ D_{i_{n}}^{(k)}$, with

$$
D_{i_{s}}^{(k)}=\left(\frac{\mathrm{d}}{\mathrm{d} x_{s}}\right)^{(k)} \circ \cdots \circ\left(\frac{\mathrm{d}}{\mathrm{d} x_{s}}\right)^{(k)}=\left(\left(\frac{\mathrm{d}}{\mathrm{d} x_{s}}\right)^{(k)}\right)^{\circ i_{s}},
$$

We will assume that $V \in \Gamma\left(\pi_{\infty, 0}^{*}\left(\pi^{\vee}\right)\right)$ has no horizontal component. Hence $a_{i}=0$ and

$$
\mathrm{j} V=\sum_{a=1}^{m}\left(V^{a} \frac{\partial}{\partial u^{a}}+\sum_{i=1}^{n}\left(\frac{\mathrm{d}}{\mathrm{d} x_{i}}\right)^{(k)}\left(V^{a}\right) \frac{\partial}{\partial u_{i}^{a}}+\sum_{2 \leq|I| \leq k} D_{I}^{(k)}\left(V^{a}\right) \frac{\partial}{\partial u_{I}^{a}}\right) .
$$

We call this space the space of evolutionary vector fields,

$$
\mathfrak{E v}(J Y)=\left\{V \in \Gamma\left(\pi_{\infty, 0}^{*}\left(\pi^{\vee}\right)\right): \frac{\partial V^{a}}{\partial u_{i}^{a}}=\frac{\partial V^{b}}{\partial u_{i}^{b}}, \frac{\partial V^{b}}{\partial u_{i}^{a}}=0, a \neq b\right\}
$$

where the functions $V^{a}$ are local in the sense that they depend on a finite number of derivatives of $u$.

For a first-order Lagrangian variational problem in a region $U \subseteq M$, the space of first variations of histories, $v=\delta \phi$, for a fixed $\phi \in \Gamma\left(\left.Y\right|_{U}\right)$, can be modeled as

$$
V \in\left\{V \in \Gamma\left(\left.\pi_{1,0}^{*}(\pi)\right|_{\mathcal{U}}\right): V \in \mathfrak{E v}(\mathcal{U} \subseteq J Y)\right\}
$$

where $\mathcal{U} \subseteq J Y$, is a neighborhood of the graph $\mathrm{j} \phi(U)$.

Let $v=d x_{1} \wedge \cdots \wedge d x_{n} \in \Omega^{n}(M)$ be a fixed volume $n$-form on $M$, and consider the Lagrangian density, $L=\mathrm{L} \cdot v \in \Omega^{n, 0}\left(\mathrm{~J}^{1} Y\right)$, with Lagrangian

$$
\mathrm{L}=\mathrm{L}\left(x_{i}, u^{a}, u_{i}^{a}\right) \in \Omega^{0}\left(J^{1} Y\right) .
$$

Consider the action functional on $U \subset M$,

$$
S_{U}(\phi)=\int_{U}\left(j^{1} \phi\right)^{*} L
$$

if we take the vertical derivative

$$
\begin{gathered}
\pi_{2,1}^{*}\left(\mathrm{~d}_{\mathrm{v}} L\right)=\mathrm{d}_{\mathrm{h}} \Theta_{L}+E(L) \\
\Theta_{L}=-\sum_{i=1}^{n} \sum_{a=1}^{m} \frac{\partial \mathrm{L}}{\partial u_{i}^{a}} \vartheta^{a} \wedge v_{i} \in \Omega^{n-1,1}\left(\mathrm{~J}^{1} Y\right), \quad d x_{i} \wedge v_{i}=v
\end{gathered}
$$


and $E(L)\left(x ; u^{(2)}\right)=\sum_{a=1}^{m} E_{a}(\mathrm{~L}) \cdot \vartheta^{a} \wedge v \in \Omega^{n, 1}\left(\mathrm{~J}^{2} Y\right)$, then the Euler-Lagrange equations are

$$
E_{a}(\mathrm{~L})=\frac{\partial \mathrm{L}}{\partial u^{a}}-\sum_{i=1}^{n} \frac{\mathrm{d}}{\mathrm{d} x_{i}}\left(\frac{2)}{\partial u_{i}^{a}}\right)=0, \quad a=1, \ldots, m
$$

Recall that $\left(\mathrm{d} / \mathrm{d} x_{i}\right)^{(2)}=\partial / \partial x_{i}+\sum_{b}\left(u_{i}^{b} \partial / \partial u^{b}+\sum_{j} u_{i j}^{b} \partial / \partial u_{j}^{b}\right)$. Another way of obtaining the Euler-Lagrange equations is by $E(L)=\mathrm{I}\left(\mathrm{d}_{\mathrm{v}} L\right)$ where we use the integration by parts operator $\mathrm{I}$ : $\Omega^{n, s}\left(\mathrm{~J}^{1} Y\right) \rightarrow \Omega^{n, s}\left(\mathrm{~J}^{2} Y\right)$ for $s>0$, satisfying $\left|\circ \mathrm{d}_{\mathrm{h}}=0, \mathrm{I}\right| \mathrm{I}=\mathrm{I}$ and that $\alpha-\mid \alpha$ is $\mathrm{d}_{\mathrm{h}}$-exact. In coordinates $\mathrm{I}$ it is given by

$$
\mathrm{I}(\alpha)=\frac{1}{s} \vartheta^{a} \wedge \sum_{a=1}^{m}\left[\iota \frac{\partial}{\partial u^{a}} \alpha-\sum_{j=1}^{n} \frac{\mathrm{d}}{\mathrm{d} x_{j}}\left(\iota \frac{\partial}{\partial u_{j}^{a}} \alpha\right)\right] .
$$

Meanwhile, the locus of the Euler-Lagrange PDEs

$$
\mathcal{E}_{L}:=\mathrm{j}\left\{\left(x ; u^{(2)}\right) \in \mathrm{J}^{2} Y: E(L)\left(x ; u^{(2)}\right)=0\right\} \subseteq J Y .
$$

The space of solutions of the Euler-Lagrange equations

$$
\mathcal{A}_{U}:=\left\{\phi \in \Gamma(Y): \mathrm{j} \phi(M) \subseteq \mathcal{E}_{L}\right\} .
$$

On the other hand, if we define the form $\Omega_{L}=-d_{\mathrm{v}} \Theta_{L} \in \Omega^{n-1,2}\left(J^{1} Y\right)$, or

$$
\Omega_{L}=\sum_{i=1}^{n}\left(\sum_{b, a=1}^{m}\left(\frac{\partial^{2} \mathrm{~L}}{\partial u^{b} \partial u^{a}} \vartheta^{b} \wedge \vartheta^{a}+\sum_{j=1}^{n} \frac{\partial^{2} \mathrm{~L}}{\partial u_{j}^{b} \partial u_{i}^{a}} \vartheta_{j}^{b} \wedge \vartheta^{a}\right)\right) \wedge v_{i} .
$$

For a first variation $\delta \phi$ modeled by $V \in \mathfrak{E} \mathfrak{v}\left(\left.J Y\right|_{U}\right)$, let us consider the Cartan formula for vertical derivation

$$
\mathcal{L}_{V} b=\mathrm{d}_{\mathrm{v}}\left(\iota_{\mathrm{j} V} b\right)+\iota_{\mathrm{j} V} \mathrm{~d}_{\mathrm{v}} b
$$

see [18] Proposition 1.16. Then

$$
\pi_{2,1}^{*}\left(\mathcal{L}_{\mathrm{j} V} b\right)=d\left(\pi_{2,1}^{*}\left(\iota_{\mathrm{j} 1} b\right)\right)+\iota_{\mathrm{j} 1 V}\left(d\left(\pi_{2,1}^{*} b\right)\right)
$$

Therefore,

$$
\begin{aligned}
& \mathrm{d}_{\mathrm{h}}\left(\iota_{\mathrm{j} 1} V^{1} b\right)=\left(d \circ \pi_{2,1}^{*}-\pi_{2,1}^{*} \circ \mathrm{d}_{\mathrm{v}}\right)\left(\iota_{\mathrm{j} 1} V\right)= \\
& -\left(\iota_{\mathrm{j} 2} V\left(d \circ \pi_{2,1}^{*}-\pi_{2,1}^{*} \circ \mathrm{d}_{\mathrm{v}}\right)\right) b=-\iota_{\mathrm{j} 2} V\left(\mathrm{~d}_{\mathrm{h}} b\right)
\end{aligned}
$$

or

$$
\mathrm{d}_{\mathrm{h}}\left(\iota_{\mathrm{j}^{1} V}(\cdot)\right)=-\iota_{\mathrm{j} 2} V\left(\mathrm{~d}_{\mathrm{h}}(\cdot)\right)
$$

In particular $d_{h}\left(\iota_{j^{1} V} \Theta_{L}\right)=-l_{j^{2} V}\left(d_{h} \Theta_{L}\right)$. Hence the variation for the action is

$$
\begin{gathered}
\left.\frac{d}{d \varepsilon}\right|_{\varepsilon=0} S_{U}\left(\phi_{\varepsilon}\right)=\left.\frac{d}{d \varepsilon}\right|_{\varepsilon=0} \int_{U}\left(j^{1} \phi_{\varepsilon}\right)^{*} L= \\
\int_{U} j^{1} \phi^{*}\left(\mathcal{L}_{\mathrm{j}^{1} V} L\right)=\int_{U} \mathrm{j}^{1} \phi^{*}\left(\iota_{\mathrm{j}^{1} V} \mathrm{~d}_{\mathrm{v}} L\right)= \\
\int_{U} \mathrm{j}^{2} \phi^{*}\left(\iota_{\mathrm{j} 2}{ }^{2} \pi_{2,1}^{*}\left(\mathrm{~d}_{\mathrm{v}} L\right)\right)= \\
\int_{U} \mathrm{j}^{2} \phi^{*}\left(\iota_{j^{2} X} \mathrm{~d}_{\mathrm{h}} \Theta_{L}+\iota_{\mathrm{j}^{2} V} E(L)\right)=
\end{gathered}
$$




$$
\begin{gathered}
-\int_{U} \mathrm{j}^{2} \phi^{*}\left(\mathrm{~d}_{\mathrm{h}}+\pi_{2,1}^{*} \circ \mathrm{d}_{\mathrm{v}}\right)\left(\iota_{\mathrm{j} 1} \Theta_{L}\right)+\int_{U} \mathrm{j}^{1} \phi^{*}\left(\iota_{\mathrm{j} 2} V(L)\right) \\
+\int_{U} \mathrm{j}^{1} \phi^{*}\left(\mathrm{~d}_{\mathrm{v}}\left(\iota_{\mathrm{j} 1} \Theta_{L}\right)\right) \\
=-\int_{U} \mathrm{j}^{2} \phi^{*} d\left(\iota_{\mathrm{j} 1} \Theta_{L}\right)+\int_{U} \mathrm{j}^{2} \phi^{*}\left(\iota_{\mathrm{j} 2 V} E(L)\right) \\
=-\int_{\partial U} \mathrm{j}^{1} \phi^{*}\left(\iota_{\mathrm{j} 1} \Theta \Theta_{L}\right)+\int_{U} \mathrm{j}^{2} \phi^{*}\left(\iota_{\mathrm{j} 2} V(L)\right) .
\end{gathered}
$$

Proposition A1. Let $\hat{\Theta}_{L}=-\Theta_{L}+L$ be for the Poincarè-Cartan form, which is also the principal Lepage equivalent of $\Theta_{L}$, and let $\phi$ be a section. The following assertions are equivalent

1. $\phi \in \mathcal{A}_{U}$.

2. For every vertical vector field, $V \in \mathfrak{E v}\left(\left.J Y\right|_{U}\right)$, the $n$-form $(\mathrm{j} \phi)^{*} \iota_{\mathrm{j} V} \hat{\Omega}_{L}$ in $U \subseteq M$, vanishes.

3. The Euler-Lagrange equations hold for every $x \in U$

$$
\frac{\partial \mathrm{L}}{\partial u^{a}}(\mathrm{j} \phi(x))-\sum_{i=1}^{n} \frac{\partial^{2} \mathrm{~L}}{\partial x_{i} \partial u_{i}^{a}}(\mathrm{j} \phi(x))=0, \quad \forall a=1, \ldots, m .
$$

Notice that in the Euler-Lagrange equations $E_{a}(\mathrm{~L})=0$ arising from $\mathrm{j}^{2} \phi^{*} E(L)=0$, the total horizontal derivations $\mathrm{d} / \mathrm{d} x_{i}$ are involved. Meanwhile, the Euler-Lagrange equations mentioned in Proposition A1 deal with partial horizontal derivations, $\partial / \partial x_{i}$, see $[1,14]$.

In general for an $(n-1)$-dimensional manifold $\Sigma \subseteq U$, we can define the 1-form

$$
\left(\theta_{L \Sigma}\right)_{\phi}(v):=\int_{\Sigma} \mathrm{j} \phi^{*}\left(\iota_{\mathrm{j} V} \Theta_{L}\right), \quad \forall \phi, v=\delta \phi,
$$

where the variation $v=\delta \phi$ corresponds to $V \in \mathfrak{E} \mathfrak{v}\left(\left.J Y\right|_{U}\right)$. For $\phi \in \mathcal{A}_{U}$,

$$
\left(\left.\mathrm{d} S_{U}\right|_{\mathcal{A}_{U}}\right)_{\phi}(v)=-\left(\left.\theta_{L, \partial U}\right|_{\mathcal{A}_{U}}\right)_{\phi}(v)
$$

Define the presymplectic structure $\omega_{L \Sigma}:=-\mathrm{d} \theta_{L \Sigma}, \forall \phi$, so that $\forall v=\delta \phi, v^{\prime}=(\delta \phi)^{\prime}$ we have

$$
\left(\omega_{L \Sigma}\right)_{\phi}\left(v, v^{\prime}\right)=\int_{\Sigma} \mathrm{j} \phi^{*}\left(\iota_{\mathrm{j} V^{\prime}} \mathrm{l}_{\mathrm{j} V} \Omega_{L}\right) .
$$

From $d_{v} \Omega_{L}=0$ it follows that $\mathrm{d} \omega_{L \Sigma}=0$.

\section{References}

1. Goldschmidt, H.; Sternberg, S. The Hamilton-Cartan formalism in the calculus of variations. Annales de l'Institut Fourier 1973, 23, 203-267. [CrossRef]

2. Kijowski, J. A finite-dimensional canonical formalism in the classical field theory. Commun. Math. Phys. 1973, 30, 99-128. [CrossRef]

3. Deligne, P.; Freed, D.S. Classical field theory. In Quantum Fields and Strings: A Course for Mathematicians; American Mathematical Society: Providence, RI, USA; Princeton, NJ, USA, 1999; Volume 1, Chapter 1, pp. 137-225.

4. Lunev, F.A. An analogue of the Noether theorem for non-Noether and nonlocal symmetries. Akademiya Nauk SSSR Teoreticheskaya i Matematicheskaya Fizika 1990, 84, 205-210.

5. Anco, S.C.; Bluman, G. Derivation of conservation laws from nonlocal symmetries of differential equations. J. Math. Phys. 1996, 37, 2361-2375. [CrossRef]

6. Olver, P.J.; Sanders, J.A.; Wang, J.P. Ghost symmetries. J. Nonlinear Math. Phys. 2002, 9 (Suppl. 1), 164-172. [CrossRef] 
7. Zapata, J.A. Gauge from Holography and Holographic Gravitational Observables. Adv. High Energy Phys. 2019, 2019. [CrossRef]

8. Sanders, K.; Dappiaggi, C.; Hack, T.P. Electromagnetism, local covariance, the Aharonov-Bohm effect and Gauss' law. Commun. Math. Phys. 2014, 328, 625-667. [CrossRef]

9. Reyes, E.G. On covariant phase space and the variational bicomplex. Int. J. Theor. Phys. 2004, 43, 1267-1286. [CrossRef]

10. Vitagliano, L. Secondary calculus and the covariant phase space. J. Geom. Phys. 2009, 59, 426-447. [CrossRef]

11. Zuckerman, G.J. Action principles and global geometry. In Proceedings of the Conference on Mathematical Aspects of String Theory, San Diego, CA, USA, 21 July-2 August 1986; World Science Publishing: Singapore, 1986; Volume 1.

12. Hélein, F. Multisymplectic formulation of Yang-Mills equations and Ehresmann connections. Adv. Theor. Math. Phys. 2015, 19, 805-835. [CrossRef]

13. Hélein, F.; Kouneiher, J. The notion of observable in the covariant Hamiltonian formalism for the calculus of variations with several variables. Adv. Theor. Math. Phys. 2004, 8, 735-777. [CrossRef]

14. Forger, M.; Romero, S.V. Covariant poisson brackets in geometric field theory. Commun. Math. Phys. 2005, 256, 375-410. [CrossRef]

15. Díaz-Marín, H.G.; Oeckl, R. Quantum abelian Yang-Mills theory on Riemannian manifolds with boundary. SIGMA Symmetry Integr. Geom. Methods Appl. 2018, 14, Paper No. 105, 31. [CrossRef]

16. Arnold, V.I.; Khesin, B.A. Topological Methods in Hydrodynamics; Springer: New York, NY, USA, 1998; Volume 125.

17. Khesin, B.; Wendt, R. The Geometry of Infinite-Dimensional Groups; Springer: Berlin, Germany, 2009; Volume 51.

18. Anderson, I.M. The Variational Bicomplex. Book Draft, Free Web Version Edition, 2004. Available online: https:/ / ncatlab.org/nlab / files / AndersonVariationalBicomplex.pdf (accessed on 7 June 2019).

19. Schwarz, G. Hodge Decomposition-A Method for Solving Boundary Value Problems; Springer: Berlin, Germany, 1995; Volume 1607.

20. Krasilshchik, I.S.; Vinogradov, A.M. Symmetries and Conservation Laws for Differential Equations of Mathematical Physics; Translations of Mathematical Monographs, AMS, Draft Edition; American Mathematical Society: Providence, RI, USA, 1999.

21. Cattaneo, A.S.; Mnev, P.; Reshetikhin, N. Semiclassical quantization of classical field theories. In Mathematical Aspects of Quantum Field Theories; Calaque, D., Strobl, T., Eds.; Mathematical Physics Studies, Chapter Part III: (Semi-)Classical Field Theories; Springer: Cham, Switerland, 2015; pp. 275-314.

22. Sengupta, A.N. The moduli space of flat connections on oriented surfaces with boundary. J. Funct. Anal. 2002, 190, 179-232. [CrossRef]

23. Díaz-Marín, H.G.; Zapata, J.A. Observable currents and a covariant poisson algebra of physical observables. arXiv 2018, arXiv:1704.07955.

24. Krupka, D. Introduction to Global Variational Geometry; Atlantis Press: Paris, France, 2015; Volume 1.

25. Olver, P.J. Applications of Lie Groups to Differential Equations; Springer: New York, NY, USA, 1986; Volume 107.

(C) 2019 by the author. Licensee MDPI, Basel, Switzerland. This article is an open access article distributed under the terms and conditions of the Creative Commons Attribution (CC BY) license (http:/ / creativecommons.org/licenses/by/4.0/). 